PACIFIC JOURNAL OF MATHEMATICS

Vol. 178, No. 1, 1997

\title{
A POLYHEDRAL TRANSVERSALITY THEOREM FOR ONE-PARAMETER FIXED POINT THEORY
}

\author{
Thomas PlavchaK
}

\begin{abstract}
The fixed point set of a piecewise linear $(P L) \operatorname{map} h: P \times I \rightarrow$ $P$ is the set of points where $h$ coincides with the projection $\pi: P \times I \rightarrow P$; it is denoted by $\mathbf{F i x}(h)$ and is a subpolyhedron of $P \times I$. When $P$ is a compact polyhedron, we show how to deform $h$ (with appropriate control) to a new $P L$ map $h^{\prime}$ so that $\mathbf{F i x}\left(h^{\prime}\right)$ is as nice as possible. Indeed it is not hard to arrange that $\operatorname{Fix}\left(h^{\prime}\right)$ have dimension $\leq 1$ (Theorem A), but one would wish for a map $h^{\prime}$ such that Fix $\left(h^{\prime}\right)$ is a manifold of dimension $\leq 1$. This is achieved in Theorem B. If $P$ is a $P L$ manifold, Theorem $\mathrm{B}$ reduces to a standard $P L$ transversality theorem (Theorem C).
\end{abstract}

\section{Introduction.}

In recent years there has been considerable interest in one-parameter fixed point theory. See, for example, $\left[\mathbf{D}_{1}\right],\left[\mathbf{D}_{2}\right],[\mathbf{D G}],\left[\mathbf{G N}_{1-5}\right],[\mathbf{G N O}],[\mathbf{J}]$. A basic requirement in using that theory is a "preparation theorem" of the kind described in the abstract. Indeed, several of the papers mentioned cite the present paper.

To state our theorems precisely we must set up our simplicial notation. By a simplex of a finite simplicial complex $K$ in $\mathbb{R}^{n}$ we mean an "open simplex"; thus a $k$-simplex $t$ is an open subset of a $k$-dimensional affine subspace of $\mathbb{R}^{n}$. If $t$ is a simplex, $\operatorname{cl}(t)$ is its closure in $\mathbb{R}^{n}$ and $\operatorname{bd}(t)$ is its boundary. The subspace of $\mathbb{R}^{n}$ spanned by $t$ is denoted by $\operatorname{span}(t)$. If the simplex $s$ is a face of a simplex $t$ we write $s<t$; in particular $t<t$. We note that since our simplexes are open, if $s<t$ and $s \neq t$ then $s \cap t=\emptyset$. The simplicial complexes $\{s \mid s<t\}$ and $\{s \mid s<t$ and $s \neq t\}$ are denoted by $\bar{t}$ and $\partial t$ respectively. The barycenter of $s$ is denoted by $b(s)$. When the vertices $v_{0}, \ldots, v_{n}$ of $K$ span a simplex of $K$ we sometimes denote that simplex by $\left\langle v_{0}, \cdots, v_{n}\right\rangle$ (except that we sometimes write $v$ rather than $\langle v\rangle$ for a 0 -simplex); when $s$ and $t$ are faces of $\left\langle v_{0}, \ldots, v_{n}\right\rangle$, the face spanned by the vertices of $s$ and of $t$ is the join of $s$ and $t$, denoted $s * t$. If $s \in K$, the star of $s$ in $K$ is $s t(s, K)=\{t \in K \mid s<t\}$ and the link of $s$ in $K$ is $l k(s, K)=\{t \in K \mid s$ and $t$ have no vertex in common and $s * t \in K\}$. If 
$M \subset K,|M|=\cup\{s \mid s \in M\}$. If $f: K \rightarrow L$ is a simplicial map (between finite simplicial complexes in Euclidean spaces), $|f|:|K| \rightarrow|L|$ denotes the corresponding "simplicial map" of spaces.

Throughout the paper we make some Standing Assumptions: $K$ is a finite simplicial complex with $|K| \subset \mathbb{R}^{n}, K_{0}$ is a simplicial subdivision of $K \times I$ formed without adding any new vertices, $K_{0}^{\prime}$ is a subdivison of a barycentric subdivision of $K_{0}$, and $f: K_{0}^{\prime} \rightarrow K$ is a simplicial map. By simplicial approximation, any map $|K| \times I \rightarrow|K|$ is homotopic to a map with these properties. For the construction of $K_{0}$ see [RS, page 16]. $|\pi|:\left|K_{0}^{\prime}\right| \rightarrow|K|$ denotes the projection $(x, t) \mapsto x$. We note that $\pi: K_{0} \rightarrow K$ is simplicial and that $\operatorname{Fix}(|f|)=\left\{x \in\left|K_{0}\right||| f|(x)=| \pi \mid(x)\right\}$.

Theorem A. $\operatorname{dim}(\operatorname{Fix}(|f|)) \leq 1$. More precisely: Let $t$ be a p-simplex of $K_{0}^{\prime}$ containing a fixed point of $|f|$ and let $s=f(t)$; then $\operatorname{dim}(s)=p$ or $p-1$.

(i) If $\operatorname{dim}(s)=p, t$ contains exactly one fixed point.

(ii) If $\operatorname{dim}(s)=p-1$, then $\operatorname{Fix}(|f|) \cap t$ is an open line segment in the boundary of which is fixed and contained in $\mathrm{bd}(t)$.

(iii) If $t \subset|K| \times\{0,1\}$, then $t$ contains exactly one fixed point.

To state our next theorem we need some notation.

If $s \in K$, we write $M_{s}=|\pi|^{-1}(\operatorname{cl}(s))$. This is a closed cell of the product cell complex $K \times I$ and hence it is subdivided as a subcomplex of any simplicial subdivision of $K \times I$. We write $M_{s}^{\prime}$ for the subcomplex of $K_{0}^{\prime}$ such that $M_{s}=\left|M_{s}^{\prime}\right|$. Similarly (below) $M_{s}^{\prime \prime}$ corresponds to $K_{0}^{\prime \prime}$ etc. The space $M_{s}$ is a closed ball of dimension $\operatorname{dim}(s)+1$, so $M_{s}^{\prime}, M_{s}^{\prime \prime}$ etc. are pseudomanifolds of that dimension, and $\partial M_{s}$ is triangulated by $\partial M_{s}^{\prime}, \partial M_{s}^{\prime \prime}$ etc. Note that if $t \in M_{s}^{\prime}$ and $\operatorname{dim}(t)=\operatorname{dim}(s)$ then $l k\left(t, M_{s}^{\prime}\right)$ consist of at most two vertices. For $i=0$ or $1, K_{0, i}^{\prime}$ denotes the subcomplex of $K_{0}^{\prime}$ triangulating $|K| \times\{i\}$.

Let $N \subset\left|K_{0}^{\prime}\right|$ be the union of the stars of all (open) simplexes which meet $\operatorname{Fix}(|f|)$. Let $\mathcal{U}$ be the cover of $|K|$ by the closures of the stars of vertices of $K$. Now we can state our main theorem: qualitatively, it says that the fixed point set can be made as nice as could be expected; see Figure 1 .

Theorem B. There is a subdivision $K_{0}^{\prime \prime}$ of $K_{0}^{\prime}$ and a simplicial map $g$ : $K_{0}^{\prime \prime} \rightarrow K$, with $|g| \mathcal{U}$-homotopic to $|f|$ by a homotopy rel $\operatorname{cl}\left(\left|K_{0}^{\prime}\right|-N\right)$, such that

(i) $\operatorname{Fix}(|g|) \subset S_{0} \cup S_{1}$ where $S_{1}=|\pi|^{-1}(\cup\{s \in K \mid l k(s, K)=\emptyset\})$, $S_{0}=|\pi|^{-1}(\cup\{s \in K \mid l k(s, K) \neq \emptyset$ but not connected $\})$, and $\operatorname{dim}\left(S_{i} \cap\right.$ $\operatorname{Fix}(|g|))=i$ for $i=0$ and 1 ,

(ii) $\operatorname{Fix}(|g|)$ is a 1-manifold placed in the following way with respect to the simplexes of $K_{0}^{\prime \prime}:$ For $t \in K_{0}^{\prime \prime}$ and $s \in K$ such that $|\pi|(t) \subset s$ and $t \cap \operatorname{Fix}(|g|) \neq \emptyset$, 
(a) $\quad$ if $l k(s, K)=\emptyset, \operatorname{dim}(t)=\operatorname{dim}(s)$ and $t \in \partial M_{s}^{\prime \prime}$, then $\left|\operatorname{st}\left(t, K_{0}^{\prime \prime}\right)\right| \cap$ $\operatorname{Fix}(|g|)$ is a half-open line segment with an endpoint in $t$;

(b) if $l k(s, K)=\emptyset$, and $\operatorname{dim}(t)=\operatorname{dim}(s)$ or $\operatorname{dim}(s)+1$ and $t \notin$ $\partial M_{s}^{\prime \prime}$, then $\left|s t\left(t, K_{0}^{\prime \prime}\right)\right| \cap \operatorname{Fix}(|g|)$ is the union of two half-open line segments;

(c) if $l k(s, K) \neq \emptyset$, then $l k(s, K)$ is not connected, $t$ contains just one fixed point of $|g|$, and $\left|s t\left(t, K_{0}^{\prime \prime}\right)\right| \cap \operatorname{Fix}(|g|)$ is the union of two half-open line segments.

Moreover, if for $i=0$ and 1 the points of $(\mathrm{Fix}|f|) \cap\left|K_{0, i}^{\prime}\right|$ all lie in principal simplexes of $K_{0, i}^{\prime}$ then the homotopy from $|f|$ to $|g|$ can be chosen to be rel $|K| \times\{0,1\}$ and $K_{0}^{\prime \prime}$ can be chosen to have $K_{0,0}^{\prime}$ and $K_{0,1}^{\prime}$ as subcomplexes.

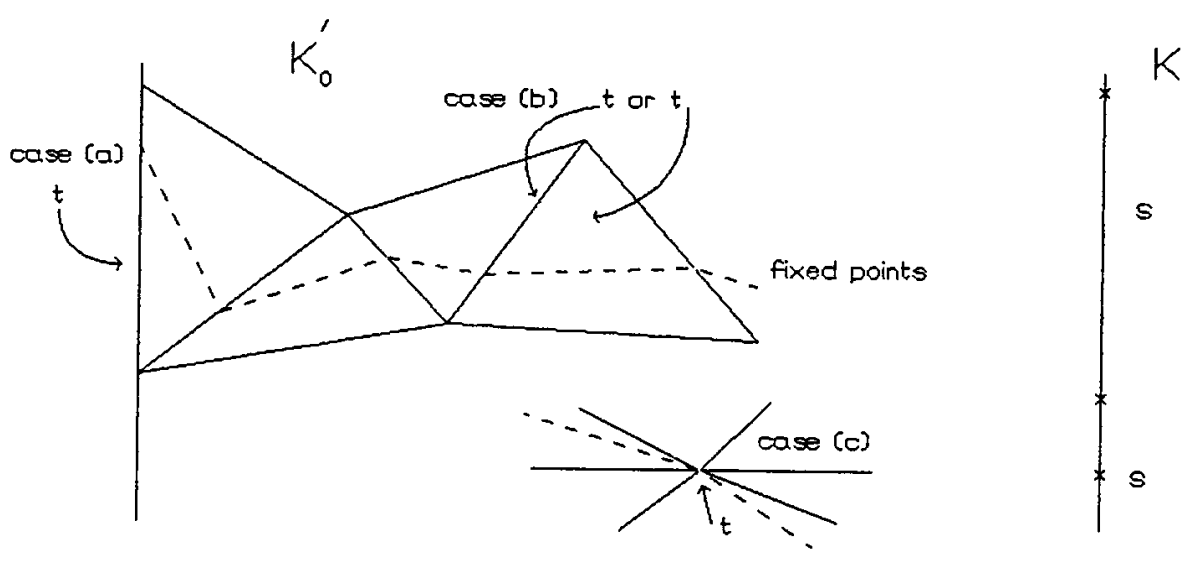

Figure 1.

Next, we recall the definition of $P L$ manifold transversality. Let $X$ be a PL manifold and let $h: X \times I \rightarrow X$ be a PL map. We denote the graph of $h$ by $\Gamma_{h}$. We say $h$ is transverse to $|\pi|: X \times I \rightarrow X$ if $h$ has no fixed points on $(\partial X) \times I$ and each point of $\Gamma_{|\pi|} \cap \Gamma_{h}$ has a regular neighborhood in $\left(X \times I \times X, \Gamma_{|\pi|}, \Gamma_{h}, \Gamma_{|\pi|} \cap \Gamma_{h}\right)$ which is $P L$ homeomorphic to a regular neighborhood of 0 in either $\left(\mathbb{R}^{n} \times \mathbb{R} \times \mathbb{R}^{n} ; \mathbb{R}^{n} \times \mathbb{R} \times 0,0 \times \mathbb{R} \times \mathbb{R}^{n}, 0 \times \mathbb{R} \times 0\right)$ or $\left(\mathbb{R}^{n} \times \mathbb{R}_{+} \times \mathbb{R}^{n} ; \mathbb{R}^{n} \times \mathbb{R}_{+} \times 0,0 \times \mathbb{R}_{+} \times \mathbb{R}^{n}, 0 \times \mathbb{R}_{+} \times 0\right)$.

Theorem C. If $|K|$ is a PL manifold then the proof of Theorem B yields a map $|g|$ which is transverse to $|\pi|$. 


\section{Proof of Theorem A.}

Lemma 2.1 is used to locate fixed points, only in Lemma 2.1 are simplexes closed.

Lemma 2.1. Let $A$ and $B$ be closed simplexes with $\operatorname{dim}(A) \geq \operatorname{dim}(B)$ and let $f: A \rightarrow B$ be a linear extension of a map of the vertices of $A$ onto the vertices of $B$. If $g: A \rightarrow B$ is a continuous function, then there is a point $x \in A$ such that $f(x)=g(x)$.

Proof. First, assume $\operatorname{dim}(A)=\operatorname{dim}(B)$. Then $f$ is a homeomorphism and so $g \circ f^{-1}: B \rightarrow B$ is defined and continuous. By the Brouwer Fixed Point Theorem there is a point $b \in B$ such that $g \circ f^{-1}(b)=b$; let $x=f^{-1}(b)$. Then $f(x)=b=g \circ f^{-1}(b)=g(x)$.

If $\operatorname{dim}(A)>\operatorname{dim}(B)$, then there is a face $C$ of $A$ such that $f(C)=B$ and $\operatorname{dim}(C)=\operatorname{dim}(B)$. Thus there is a point $x$ in $C$ such that $f(x)=$ $g(x)$.

Lemma 2.2. Let $t$ be a $p$-simplex in $\mathbb{R}^{n}, p \geq 1, v$ a vertex of $t$, and $l$ a line in $\mathbb{R}^{n}$. If $l \cap t$ contains at least two points, then there is a proper face $t^{\prime}$ of $t$ having $v$ as a vertex and such that $t^{\prime} \cap l \neq \emptyset$.

Proof. Because $l$ meets $t$ in at least two points, $l \subset \operatorname{span}(t)$; therefore $l \cap \operatorname{bd}(t)$ contains at least two points. Let $s$ be the face of $t$ opposite $v$. If no proper face of $t$ having $v$ as a vertex meets $l$, then every point in $l \cap \mathrm{bd}(t)$ is either in $s$ or a face of $s$. So $l \subset \operatorname{span}(s)$, but $l \subset \operatorname{span}(s)$ contradicts $\operatorname{span}(s) \cap t=$ $\emptyset$.

Lemma 2.3. Let $t$ be a $p$-simplex in $\mathbb{R}^{n}, p \geq 2, v$ a vertex of $t$, and $P$ a plane in $\mathbb{R}^{n}$. If $P \cap t$ contains at least three non-collinear points, then there is a face $t^{\prime}$ of $t$ such that $v$ is a vertex of $t^{\prime}, \operatorname{dim}\left(t^{\prime}\right) \leq p-2$, and $t^{\prime} \cap P \neq \emptyset$.

Proof. Let $p_{0}, p_{1}$, and $p_{2}$ in $P \cap t$ be three non-collinear points. The line $l_{0,1}$ spanned by $p_{0}$ and $p_{1}$ meets $\mathrm{bd}(t)$ at two points $x_{0}$ and $x_{1}$, and the line $l_{0,2}$ meets $\mathrm{bd}(t)$ at some point $x_{3}$. The point $x_{3}$ is not on the line spanned by $x_{0}$ and $x_{1}$ because $p_{2} \notin l_{0,1}$. Thus there are three non-collinear points of $P$ in $\operatorname{bd}(t)$.

Let $s$ be the face of $t$ opposite $v$. If no proper face of $t$ having $v$ as a vertex meets $P$, then every point in $P \cap \operatorname{bd}(t)$ is either in $s$ or a face of $s$. So $P \subset \operatorname{span}(s)$, contradicting $\operatorname{span}(s) \cap t=\emptyset$. Thus $P$ meets a proper face $t_{1}$ of $t$ having $v$ as a vertex.

Assume $\operatorname{dim}\left(t_{1}\right)=p-1$. Now $\operatorname{dim}\left(P \cup \operatorname{span}\left(t_{1}\right)\right)=\operatorname{dim}(P)+\operatorname{dim}\left(\operatorname{span}\left(t_{1}\right)\right)$ $-\operatorname{dim}\left(P \cap \operatorname{span}\left(t_{1}\right)\right)$. Since $\operatorname{dim}\left(P \cup \operatorname{span}\left(t_{1}\right)\right) \leq p$ and $\operatorname{dim}(P)=2, \operatorname{dim}(P \cap$ 
$\left.\operatorname{span}\left(t_{1}\right)\right) \geq 1$. Thus there is a line $l$ lying in $P$ and meeting $t_{1}$ in at least two points. By Lemma 2.2 there is a proper face $t^{\prime}$ of $t_{1}$ having $v$ as a vertex and such that $l \cap t^{\prime} \neq \emptyset$. Thus there is a face $t^{\prime}$ of $t$ having $v$ as a vertex such that $\operatorname{dim}\left(t^{\prime}\right) \leq p-2$ and $t^{\prime} \cap P \neq \emptyset$.

Lemma 2.4. If $t$ is a simplex of $K_{0}^{\prime}$ and $|\pi|(t)$ is contained in the simplex $s$ of $K$, then there is a vertex $v$ of $t$ such that $|\pi|(v) \in s$.

Proof. Let $K_{0}^{1}$ be the first barycentric subdivision of $K_{0}$ and let $t^{\prime \prime} \in K_{0}^{1}$ and $t^{\prime} \in K_{0}$ be such that $t \subset t^{\prime \prime} \subset t^{\prime}$. Since $s$ and $\pi\left(t^{\prime}\right)$ are simplexes of $K$ and both contain $|\pi|(t), s=\pi\left(t^{\prime}\right)$. Since $\operatorname{bd}(t) \subset \operatorname{cl}\left(t^{\prime \prime}\right)$, either $t^{\prime \prime}$, and so $t^{\prime}$, contains a vertex of $t$ or each vertex of $t$ is in $\operatorname{bd}\left(t^{\prime \prime}\right)$. If $t^{\prime}$ contains a vertex $v$ of $t$, then $|\pi|(v) \in s$.

Suppose each vertex of $t$ is in $\operatorname{bd}\left(t^{\prime \prime}\right)$. Since $t^{\prime \prime} \in K_{0}^{1}, t^{\prime \prime}=\left\langle b\left(s_{0}\right), \ldots, b\left(s_{p}\right)\right\rangle$ where $s_{0}, \ldots, s_{p} \in K_{0}$ and $s_{0}<\cdots<s_{p}$. Now $t^{\prime \prime} \subset s_{p}$ so $t \subset s_{p}$, thus $t^{\prime}=s_{p}$. Let $v^{\prime}=b\left(t^{\prime}\right)$. Let $\tau$ be the face of $t^{\prime \prime}$ opposite $v^{\prime}$. Now if each vertex of $t$ is contained in $\tau$ or a face of $\tau$, then $t \subset \operatorname{cl}(\tau)$, but $\operatorname{cl}(\tau) \cap t^{\prime \prime}=\emptyset$ contradicting $t \subset t^{\prime \prime}$. Thus there is a proper face $t_{0}$ of $t^{\prime \prime}$ having $v^{\prime}$ as a vertex and containing a vertex $v$ of $t$. Because $v^{\prime}=b\left(t^{\prime}\right), t_{0} \subset t^{\prime}$. Now $v \in t_{0} \subset t^{\prime}$ implies $|\pi|(v) \in|\pi|\left(t^{\prime}\right)$ and $|\pi|\left(t^{\prime}\right)$ is $s$.

Lemma 2.5. Let $t$ be a simplex of $K_{0}^{\prime}, s \in K,|\pi|(t) \subset s$, and let $v$ be a vertex of $t$ such that $|\pi|(v) \in s$. If $t^{\prime}$ is a face of $t$ and $v$ is a vertex of $t^{\prime}$, then $|\pi|\left(t^{\prime}\right) \subset s$.

Proof. (By induction on $\operatorname{dim}\left(t^{\prime}\right)$.) Let $t^{\prime}=\left\langle v, v_{1}, \ldots, v_{n}\right\rangle$ and let $p \in t^{\prime}$. Now $p=a v_{n}+b x$ where $x$ is a point in $\left\langle v, v_{1}, \ldots, v_{n-1}\right\rangle$. By the inductive hypothesis $|\pi|\left(\left\langle v, v_{1}, \ldots, v_{n-1}\right\rangle\right) \subset s$. Since $|\pi|(t) \subset s,|\pi|\left(v_{n}\right) \in \operatorname{cl}(s)$. Thus $|\pi|(p) \in s$.

Proof of Theorem A. Since $t$ contains a fixed point, $|\pi|(t) \subset s$. Since $f$ is simplicial, $\operatorname{dim}(f(t)) \leq \operatorname{dim}(t)$. So $\operatorname{dim}(s) \leq \operatorname{dim}(t)$. Because $|\pi|(t) \subset s$, $t \in M_{s}^{\prime}$. So $\operatorname{dim}(t) \leq \operatorname{dim}(s)+1$.

First, assume $\operatorname{dim}(s)=p$ and $x_{0}, x_{1}$ are fixed points of $|f|$ in $t$. Let $v$ be a vertex of $t$ such that $|\pi|(v) \in s$ (see Lemma 2.4). Now if $t^{\prime}$ is a proper face of $t$ having $v$ as a vertex, then $t^{\prime}$ cannot contain a fixed point of $|f|$ because if $v$ is a vertex of $t^{\prime}$ then $|\pi|\left(t^{\prime}\right) \subset s$, and if $\operatorname{dim}\left(t^{\prime}\right)<\operatorname{dim}(t)$, then $f\left(t^{\prime}\right) \neq s$. But by Lemma 2.2 the line $l$ determined by $x_{0}$ and $x_{1}$ intersects a proper face of $t$ having $v$ as a vertex and since $l \cap \operatorname{cl}(t)$ is fixed by $|f|$ this face contains a fixed point of $|f|$. This contradicts the fact that no proper face of $t$ having $v$ as a vertex can contain a fixed point.

Next, assume $\operatorname{dim}(s)=p-1$. Then there is a proper face $t_{0}$ of $t$ such that $f\left(t_{0}\right)=s$. Since $|\pi|\left(\operatorname{cl}\left(t_{0}\right)\right) \subset \operatorname{cl}(s)$, a proper face of $t$ contains a fixed point. 
So $t$ contains a line segment $l$ of fixed points the boundary of which is fixed and contained in $\operatorname{bd}(t)$. Suppose $x$ is a fixed point of $|f|$ and $x$ is not on $l$. Let $v$ be a vertex of $t$ such that $|\pi|(v) \in s$. Now if $t_{0}$ is a $(p-2)$-face of $t$ having $v$ as a vertex, then $t_{0}$ cannot contain a fixed point of $|f|$, because if $v$ a vertex of $t_{0}$ then $|\pi|\left(t_{0}\right) \subset s$, and if $\operatorname{dim}\left(t_{0}\right)=p-2$ then $f\left(t_{0}\right) \neq s$. But by Lemma 2.3 the plane $P$ determined by $l$ and $x$ meets a $(p-2)$-face of $t$ having $v$ as a vertex, and since $P \cap \operatorname{cl}(t)$ is fixed by $|f|$ this face contains a fixed point of $|f|$. This contradicts the fact that no $(p-2)$-face of $t$ having $v$ as a vertex can contain a fixed point.

Now suppose $t \subset|K| \times\{0,1\}$. Since $t \subset s \times\{0,1\}, t$ is a face of a $(p+1)$ simplex contained in $s \times I$. So $p+1 \leq \operatorname{dim}(s)+1$. Because $f(t)=s, p \geq$ $\operatorname{dim}(s)$. Thus $\operatorname{dim}(s)=p$ and $t$ contains just one fixed point.

\section{Proof of Theorem B.}

Lemma 3.1. Let $t, t^{\prime} \in K_{0}^{\prime}, s, s^{\prime} \in K,|\pi|(t) \subset s$, and $|\pi|\left(t^{\prime}\right) \subset s^{\prime}$.

(a) If $t<t^{\prime}$, then $s<s^{\prime}$.

(b) If $s<s^{\prime}$ and $t * t^{\prime} \in K_{0}^{\prime}$, then $|\pi|\left(t * t^{\prime}\right) \subset s^{\prime}$.

(c) If $t * t^{\prime} \in K_{0}^{\prime}$, then $s<s^{\prime}$ or $s^{\prime}<s$.

Proof of (a). Let $A, B \in K_{0}$ be such that $t \subset A$ and $t^{\prime} \subset B$. Then $\pi(A)=$ $s$ and $\pi(B)=s^{\prime}$. Now $\left|s t\left(A, K_{0}\right)\right|$ is an open subset of $\left|K_{0}\right|,\left|s t\left(A, K_{0}\right)\right|$ contains $t$, and each point of $t$ is a limit point of $t^{\prime}$, so $B \in \operatorname{st}\left(A, K_{0}\right)$. Thus $A<B$ and so $|\pi|(A)<|\pi|(B)$, i.e., $s<s^{\prime}$.

Proof of (b). Let $A \in K_{0}$ be such that $t * t^{\prime} \subset A$. Since $t<t * t^{\prime},|\pi|(t) \subset s$, and $|\pi|\left(t * t^{\prime}\right) \subset|\pi|(A)$, by Part (a), $s<\pi(A)$. Similarly $s^{\prime}<\pi(A)$. By Lemma 2.4 there is a vertex $v$ of $t * t^{\prime}$ such that $|\pi|(v) \in \pi(A)$. But if $v$ is a vertex of $t$, then $|\pi|(v) \in \operatorname{cl}(s)$ and if $v$ is a vertex of $t^{\prime}$, then $|\pi|(v) \in \operatorname{cl}\left(s^{\prime}\right)$. Since $s<s^{\prime}$, in either case $|\pi|(v) \in \operatorname{cl}\left(s^{\prime}\right)$. Thus $|\pi|(v)$ is in the simplex $|\pi|(A)$ and in the closure of the simplex $s^{\prime}$. This means $\pi(A)<s^{\prime}$. From above, $s^{\prime}<\pi(A)$. So $\pi(A)=s^{\prime}$. Hence $|\pi|\left(t * t^{\prime}\right) \subset s^{\prime}$.

Proof of (c). Let $A, B$, and $C$ be simplexes of $K_{0}$ such that $t \subset A, t^{\prime} \subset B$, and $t * t^{\prime} \subset C$. Since $t * t^{\prime} \subset \operatorname{cl}(C), t \subset \operatorname{cl}(C)$. So $A<C$. Similarly $B<C$. So $A * B \in K_{0}$. Since $t * t^{\prime} \subset A * B, A * B=C$.

Let $X, Y$, and $Z$ be simplexes of $K_{0}^{1}$ be such that $t \subset X, t^{\prime} \subset Y$, and $t * t^{\prime} \subset Z$. From the last paragraph $X * Y \in K_{0}^{1}$ and $X * Y=Z$. Let $X=$ $\left\langle b\left(T_{0}\right), b\left(T_{1}\right), \ldots, b\left(T_{p-1}\right), b(A)\right\rangle$ and let $Y=\left\langle b\left(S_{0}\right), b\left(S_{1}\right), \ldots, b\left(S_{q-1}\right), b(B)\right\rangle$ where $T_{0}<T_{1}<\cdots<T_{p-1}<A$ and $S_{0}<S_{1}<\cdots<S_{q-1}<B$. Now $X * Y \in K_{0}^{1}$ implies $A<B$ or $B<A$. So $\pi(A)<\pi(B)$ or $\pi(B)<\pi(A)$, that is, $s<s^{\prime}$ or $s^{\prime}<s$. 
Proposition 3.2. Let the simplex $t_{0}$ in $K_{0}^{\prime}$ contain a fixed point of $|f|$. Then each component of $\left(\operatorname{Fix}(|f|) \cap\left|s t\left(t_{0}, K_{0}^{\prime}\right)\right|\right)-t_{0}$ is contained in a simplex of $s t\left(t_{0}, K_{0}^{\prime}\right)$.

Proof. Suppose $t \in s t\left(t_{0}, K_{0}^{\prime}\right), t \neq t_{0}$, and $t$ contains a fixed point of $|f|$. Suppose that $t^{\prime}$ is a proper face of $t, t_{0}$ is a proper face of $t^{\prime}$, and $t^{\prime}$ contains a fixed point of $|f|$. Then $\mathrm{cl}(t)$ contains three non-collinear fixed points of $|f|: a, b$, and $c$. Thus the fixed point set of $|f|$ contains a 2-dimensional subset: $\operatorname{cl}(t) \cap \operatorname{span}(\{a, b, c\})$. But this contradicts Theorem A. Thus $t^{\prime}$ contains no fixed points of $|f|$.

Lemma 3.3. Let $M$ and $N$ be complexes and let $L$ be a subcomplex of $M$. Suppose $f: M \rightarrow N$ and $g: M \rightarrow N$ are simplicial maps that agree on $L$. Furthermore suppose for each $x \in|M|-|L|$ there are simplexes $s, t$, and $s_{0}$ in $N$ such that $s_{0}<s, s_{0}<t,|f|(x) \in t$, and $|g|(x) \in \operatorname{cl}(s)$. Then $|f|$ is $\mathcal{U}$-homotopic to $|g| \operatorname{rel}|L|$.

Proof. Use Lemma 1, page 124 in [Br].

Let $M$ be a simplicial complex and $L$ a subcomplex of $M$. Define the simplicial subdivision of $M$ modulo the subcomplex $L$, denoted by $M_{L}^{\prime}$, as follows: The vertices of $M_{L}^{\prime}$ are the vertices of $M$ along with the vertices $b(t)$ where $t$ is a simplex in $M-L$. For $p \geq 1$, the $p$-simplexes of $M_{L}^{\prime}$ are of the form $\left\langle v_{0}, \ldots, v_{q}, b\left(t_{q+1}\right), \ldots, b\left(t_{p}\right)\right\rangle$ where $\left\langle v_{0}, \ldots, v_{q}\right\rangle$ is a $q$-simplex in $L, t_{q+1}, \ldots, t_{p}$ are distinct simplexes in $M-L$, and $\left\langle v_{0}, \ldots, v_{q}\right\rangle \supsetneqq t_{q+1}<$ $\cdots<t_{p}$. Here $q$ can equal 0 and $p$ can equal $q$.

Proposition 3.4. Assume $t_{0}$ is a p-simplex of $K_{0}^{\prime}, t_{0}$ contains a fixed point of $|f|, f\left(t_{0}\right)=s_{0}$, and $\operatorname{dim}\left(s_{0}\right)=p$. Assume $l k\left(t_{0}, M_{s_{0}}^{\prime}\right)$ consists of two vertices $v_{1}, v_{2}$ and $f\left(v_{1}\right) \neq f\left(v_{2}\right)$ are not vertices of $s_{0}$. Let $L$ be the subcomplex of $K_{0}^{\prime}$ consisting of $t_{0}$ and all simplexes that do not have $t_{0}$ as a face. Then there is a simplicial map $|g|:\left|\left(K_{0}^{\prime}\right)_{L}^{\prime}\right| \rightarrow|K|$ homotopic to $|f|$ rel $|L|$ such that $\operatorname{Fix}(|g|) \cap\left|s t\left(t_{0},\left(K_{0}^{\prime}\right)_{L}^{\prime}\right)\right|$ is a 1-manifold.

Proof. Define $g$ on the vertices of $\left(K_{0}^{\prime}\right)_{L}^{\prime}$ as follows: If $a$ is a vertex of $L, g(a)=$ $f(a)$; if $t_{0}$ is a proper face of $t$ and $t \neq v_{i} * t_{0}$ for $i=1$ or 2 , then let $g(b(t))$ be any vertex of $s_{0}$; for $i=1$ and 2 let $g\left(b\left(v_{i} * t_{0}\right)\right)=f\left(v_{i}\right)$.

Let $\sigma=\left\langle a_{0}, a_{1}, \ldots, a_{l}, b\left(t_{l+1}\right), \ldots, b\left(t_{m}\right)\right\rangle$ be a simplex of $\left(K_{0}^{\prime}\right)_{L}^{\prime}$. By definition, $g\left(a_{j}\right)$ is a vertex of $f\left(t_{l+1}\right)$ for $0 \leq j \leq l$, and, for $l+1 \leq j \leq$ $m, g\left(b\left(t_{j}\right)\right)$ is $f\left(v_{1}\right)$ or $f\left(v_{2}\right)$ or a vertex of $s_{0}$. If for each $j, g\left(b\left(t_{j}\right)\right)<s_{0}$, then, since $s_{0}<f\left(t_{l+1}\right), g(\sigma)$ is a face of $f\left(t_{l+1}\right)$. If for some $j_{0}, g\left(b\left(t_{j_{0}}\right)\right)=$ $f\left(v_{1}\right)$ or $f\left(v_{2}\right)$, then $j_{0}=l+1$ and $t_{j_{0}}=v_{1} * t_{0}$ or $t_{j_{0}}=v_{2} * t_{0}$. So $g(\sigma)<f\left(v_{1}\right) * s_{0}$ or $g(\sigma)<f\left(v_{2}\right) * s_{0}$. For $i=1$ and $2, f\left(v_{i}\right) * s_{0}$ is a 
simplex of $K$ because $f\left(v_{i}\right) \in l k\left(s_{0}, K\right)$. Thus $g(\sigma)$ is a simplex of $K$ and so $g:\left(K_{0}^{\prime}\right)_{L}^{\prime} \rightarrow K$ is simplicial. Furthermore, since $\sigma \subset t_{m},|f|(\sigma) \subset f\left(t_{m}\right)$; because $t_{0}<t_{m}, s_{0}<f\left(t_{m}\right)$; and $g(\sigma)$ is a subset of either $\operatorname{cl}\left(s_{0}\right)$ or $\operatorname{cl}\left(f\left(v_{1}\right) * s_{0}\right)$ or $\operatorname{cl}\left(f\left(v_{2}\right) * s_{0}\right)$. By Lemma 3.3, $|g|:\left|\left(K_{0}^{\prime}\right)_{L}^{\prime}\right| \rightarrow|K|$ is $\mathcal{U}$-homotopic to $|f|$ rel $|L|$.

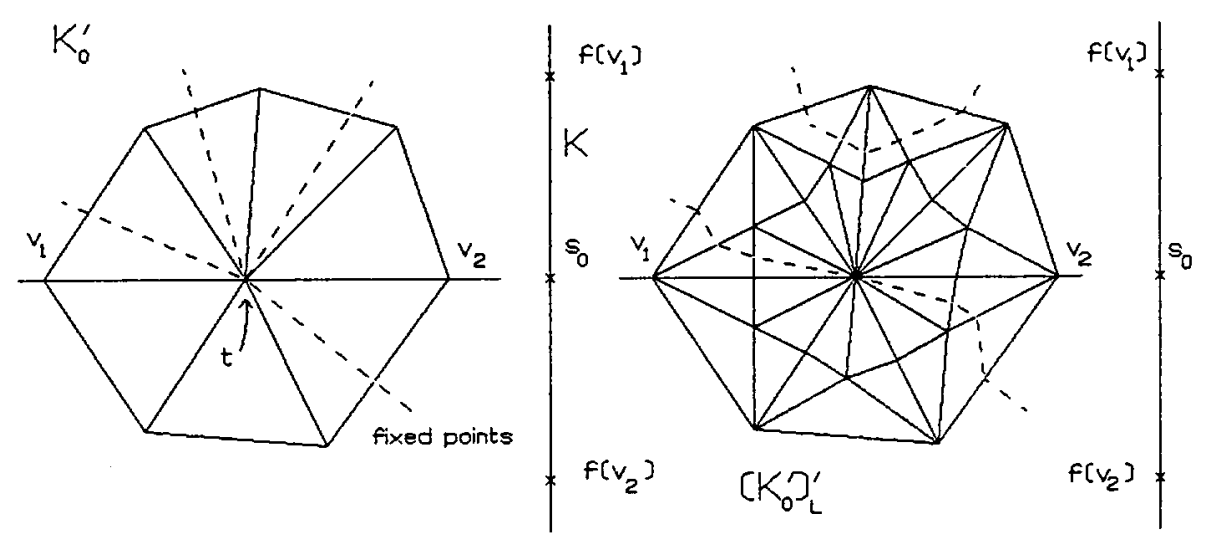

Figure 2.

Let $t_{0}=\left\langle a_{0}, \ldots, a_{p}\right\rangle$ and for $i=1$ and 2 let $s_{i}=f\left(v_{i}\right) * s_{0}$, let $\gamma_{i}$ be the unique $(p+2)$-simplex of $M_{s_{i}}^{\prime}$ having $v_{i} * t_{0}$ as a face, let $g\left(b\left(\gamma_{i}\right)\right)=f\left(a_{j_{i}}\right)$, and let $\sigma_{i}=\left\langle a_{0}, \ldots, \hat{a}_{j_{i}}, \ldots, a_{p}, b\left(v_{i} * t_{0}\right), b\left(\gamma_{i}\right)\right\rangle$. Then $g\left(\sigma_{i}\right)=s_{i}$ and $|\pi|\left(\sigma_{i}\right) \subset s_{i}$ for $i=1$ and 2. So $\operatorname{cl}\left(\sigma_{i}\right)$ contains a fixed point of $|g|$. No proper face of $\sigma_{i}$ contains a fixed point of $|g|$. [Proof: Using Theorem 1 no face of $\left\langle a_{0}, \ldots, \hat{a}_{j_{i}}, \ldots, a_{p}\right\rangle$ contains a fixed point of $|g|$. Any face of $\sigma_{i}$ having $b\left(v_{i} * t_{0}\right)$ as a vertex but not $b\left(\gamma_{i}\right)$ is projected into $s_{0}$, but the image under $|g|$ of any such face is not $s_{0}$. Any face having $b\left(\gamma_{i}\right)$ as a vertex but not $b\left(v_{i} * t_{0}\right)$ is projected into $s_{i}$, but the image under $|g|$ of such a face is a face of $s_{0}$. If $t=\left\langle a_{0}, \ldots, \hat{a}_{l}, \ldots, \hat{a}_{j_{i}}, \ldots, a_{p}, b\left(v_{i} * t_{0}\right), b\left(\gamma_{i}\right)\right\rangle$ where $0 \leq l \leq p, l \neq j_{i}$, then $|\pi|(t) \subset s_{i}$ but $g(t) \neq s_{i}$.] Thus for $i=1$ and $2, \sigma_{i} * t_{0}$ is a simplex of $\left(K_{0}^{\prime}\right)_{L}^{\prime}$ and $\sigma_{i} * t_{0}$ contains a line segment of fixed points of $|g|$ with endpoints in $\sigma_{i}$ and $t_{0}$.

Let $\mu=\left\langle a_{0}, \ldots, a_{p}, b\left(t_{p+1}\right), \ldots, b\left(t_{q}\right)\right\rangle$ be a simplex in $s t\left(t_{0},\left(K_{0}^{\prime}\right)_{L}^{\prime}\right)$ and suppose $\mu$ contains a fixed point of $|g|$. Let $g(\mu)=s^{\prime}$. Because $\mu \subset t_{q}$ and $|\pi|(\mu)$ is a subset of $s^{\prime},|\pi|\left(t_{q}\right) \subset s^{\prime}$, that is, $t_{q} \in M_{s^{\prime}}^{\prime}$. If for each $j, p+1 \leq j \leq q, t_{j}$ is not the simplex $v_{1} * t_{0}$ or the simplex $v_{2} * t_{0}$, then $g(\mu)=s_{0}$. So $s^{\prime}=s_{0}$ and $t_{q} \in M_{s_{0}}^{\prime}$. But $t_{0}<t_{q}$, so $q=p$ and $\mu=t_{0}$. If for some $j_{0}, t_{j_{0}}=v_{i} * t_{0}$ for $i=1$ or 2 , then $g(\mu)=s_{i}$, so $s_{i}=s^{\prime}$ and 
$t_{q} \in M_{s_{i}}^{\prime}$. But $\gamma_{i}$ is the unique simplex in $M_{s_{i}}^{\prime}$ having $v_{i} * t_{0}$ as a proper face, thus $\mu=\sigma_{i} * t_{0}$ for $i=1$ or 2 [note that if $\mu=\left\langle a_{0}, \ldots, a_{p}, b\left(v_{i} * t_{0}\right)\right\rangle$, then $\mu$ has no fixed points because $|\pi|(\mu) \subset s_{0}$ but $\left.g(\mu)=s_{i}\right]$. Thus the only simplexes in $s t\left(t_{0},\left(K_{0}^{\prime}\right)_{L}^{\prime}\right)$ containing fixed points of $|g|$ are $\sigma_{1} * t_{0}, \sigma_{2} * t_{0}$, and $t_{0}$. Since $f\left(v_{1}\right) \neq f\left(v_{2}\right), \sigma_{1} \neq \sigma_{2}$ and so $\operatorname{Fix}(|g|) \cap\left|s t\left(t_{0},\left(K_{0}^{\prime}\right)_{L}^{\prime}\right)\right|$ is a 1-manifold.

Condition 1. A subdivision $K_{0}^{\prime \prime}$ of $K_{0}^{\prime}$, a simplicial map $g: K_{0}^{\prime \prime} \rightarrow K$, and a simplex $t_{0} \in K_{0}^{\prime \prime}$ containing a fixed point of $|g|$ satisfy Condition 1 if for each component $A$ of $\left(\operatorname{Fix}(|g|) \cap\left|s t\left(t_{0}, K_{0}^{\prime \prime}\right)\right|\right)-t_{0}$, if $t_{A} \in K_{0}^{\prime \prime}$ is the simplex of $s t\left(t_{0}, K_{0}^{\prime \prime}\right)$ that contains $A$ (see Proposition 3.2) and $v$ is a vertex in $l k\left(t_{A}, K_{0}^{\prime \prime}\right)$, then $g(v)$ is a vertex of $g\left(t_{A}\right)$.

Addendum 3.5. The simplex $t_{0}$, the subdivision $\left(K_{0}^{\prime}\right)_{L}^{\prime}$, and the map $g$ satisfy Condition 1.

Proof. Let $A$ be a component of $\left(|\operatorname{Fix}(|g|)| \cap\left|s t\left(t_{0},\left(K_{0}^{\prime}\right)_{L}^{\prime}\right)\right|\right)-t_{0}$ and let the simplex $t_{A}$ in $s t\left(t_{0},\left(K_{0}^{\prime}\right)_{L}^{\prime}\right)$ be the carrier of $A$. By construction $t_{A}=\sigma_{1} * t_{0}$ or $t_{A}=\sigma_{2} * t_{0}$. Suppose $t_{A}=\sigma_{1} * t_{0}$ and $v$ is a vertex in $l k\left(t_{A},\left(K_{0}^{\prime}\right)_{L}^{\prime}\right)$. Because $b\left(v_{1} * t_{0}\right)$ is a vertex of $\sigma_{1} * t_{0}, b\left(v_{1} * t_{0}\right)$ is not a vertex in $l k\left(t_{A},\left(K_{0}^{\prime}\right)_{L}^{\prime}\right)$. If $b\left(v_{2} * t_{0}\right) \in l k\left(t_{A},\left(K_{0}^{\prime}\right)_{L}^{\prime}\right)$, then $b\left(v_{2} * t_{0}\right) * t_{A}$ is a simplex of $\left(K_{0}^{\prime}\right)_{L}^{\prime}$. Since $b\left(v_{2} * t_{0}\right)$ is not a vertex of $t_{A}, b\left(v_{2} * t_{0}\right) * t_{A}$ is a $(p+3)$-simplex in $\left(M_{s_{1}}^{\prime}\right)_{L}^{\prime}$ but this contradicts $\operatorname{dim}\left(\left(M_{s_{1}}^{\prime}\right)_{L}^{\prime}\right)=p+2$. Thus $v \neq b\left(v_{i} * t_{0}\right)$ for $i=1$ and 2. Since $t_{0}<\sigma_{1}, v \in l k\left(t_{0},\left(K_{0}^{\prime}\right)_{L}^{\prime}\right)$. By definition of $g, g(v)$ is a vertex of $s_{0}$. Since $s_{0}<s_{1}$ and $g\left(t_{A}\right)=s_{1}, g(v)$ is a vertex of $g\left(t_{A}\right)$.

Lemma 3.6. Assume $t_{0}$ is a p-simplex of $K_{0}^{\prime}$, $t_{0}$ contains a fixed point of $|f|, f\left(t_{0}\right)=s_{0}$, and $\operatorname{dim}\left(s_{0}\right)=p$. Assume lk $\left(t_{0}, M_{s_{0}}\right)$ consists of two vertices $v_{1}$ and $v_{2}$ and $f\left(v_{1}\right) \neq f\left(v_{2}\right)$. Let $L$ be the subcomplex of $K_{0}^{\prime}$ consisting of $t_{0}$ and all simplexes that do not have $t_{0}$ as a face. If there is an arc $c_{n}$ in $l k\left(s_{0}, K\right)$ with endpoints $f\left(v_{1}\right)$ and $f\left(v_{2}\right)$ consisting of $n 1$-simplexes, then there is a simplicial map $|g|:\left|\left(K_{0}^{\prime}\right)_{L}^{\prime}\right| \rightarrow|K| \mathcal{U}$-homotopic to $|f|$ rel $|L|$ such that $l k\left(t_{0},\left(M_{s_{0}}^{\prime}\right)_{L}^{\prime}\right)$ consists of two vertices $v_{1}^{\prime}$ and $v_{2}^{\prime}$, and there is an arc $c_{n-1}$ in $l k\left(s_{0}, K\right)$ with endpoints $g\left(v_{1}^{\prime}\right)$ and $g\left(v_{2}^{\prime}\right)$ consisting of $n-11$-simplexes.

Proof. Let $c_{n}$ be the arc $\left\langle f\left(v_{1}\right)\right\rangle \cup\left\langle f\left(v_{1}\right), b_{1}\right\rangle \cup\left\langle b_{1}\right\rangle \cup \ldots \cup\left\langle b_{n-1}, f\left(v_{2}\right)\right\rangle \cup\left\langle f\left(v_{2}\right)\right\rangle$. Define $g$ on the vertices of $\left(K_{0}^{\prime}\right)_{L}^{\prime}$ as follows: if $a$ is a vertex of $L, g(a)=f(a)$; if $t$ is a simplex of $K_{0}^{\prime}$ and $t_{0}$ is a proper face of $t$ and $t \neq v_{i} * t_{0}$ for $i=1$ or 2 , then let $g(b(t))$ be any vertex of $s_{0}$; let $g\left(b\left(v_{1} * t_{0}\right)\right)=b_{1}$ and $g\left(b\left(v_{2} * t_{0}\right)\right)=f\left(v_{2}\right)$.

Let $\sigma=\left\langle a_{0}, \ldots a_{l}, b\left(t_{l+1}\right), \ldots, b\left(t_{m}\right)\right\rangle$ be a simplex of $\left(K_{0}^{\prime}\right)_{L}^{\prime}$. By definition, $g\left(a_{j}\right)<f\left(t_{l+1}\right)$ for $0 \leq j \leq l$ and, for $l+1 \leq j \leq m, g\left(b\left(t_{j}\right)\right)=b_{1}$ or $f\left(v_{2}\right)$ or is a vertex of $s_{0}$. If for each $j, g\left(b\left(t_{j}\right)\right)$ is a vertex of $s_{0}$, then, since 
$s_{0}<f\left(t_{l+1}\right), g(\sigma)$ is a face of $f\left(t_{l+1}\right)$. If for some $j_{0}, g\left(b\left(t_{j_{0}}\right)\right)=b_{1}$, then $j_{0}=l+1$ and $t_{j_{0}}=v_{1} * t_{0}$. So $g(\sigma)<\left\langle f\left(v_{1}\right), b_{1}\right\rangle * s_{0}$ because for $j>l+$ $1 g\left(b\left(t_{j}\right)\right)<s_{0}$, for $j \leq l g\left(a_{j}\right)<f\left(v_{1}\right) * s_{0}$, and $g\left(b\left(v_{1} * t_{0}\right)\right)=b_{1}$. If for some $j_{0}, g\left(b\left(t_{j_{0}}\right)\right)=f\left(v_{2}\right)$, then $j_{0}=l+1$ and $t_{j_{0}}=v_{2} * t_{0}$. So $g(\sigma)<f\left(v_{2}\right) * s_{0}$, because for $j>l+1 g\left(b\left(t_{j}\right)\right)<s_{0}$, while for $j \leq l g\left(a_{j}\right)<f\left(v_{2}\right) * s_{0}$ and $g\left(b\left(v_{2} * t_{0}\right)\right)=f\left(v_{2}\right)$. Since $f\left(v_{2}\right) \in l k\left(s_{0}, K\right)$ and $\left\langle f\left(v_{1}\right), b_{1}\right\rangle \in l k\left(s_{0}, K\right)$ in all cases $g(\sigma)$ is a simplex of $K$. So $g:\left(K_{0}^{\prime}\right)_{L}^{\prime} \rightarrow K$ is simplicial. Furthermore, since $\sigma \subset t_{m},|f|(\sigma) \subset f\left(t_{m}\right)$; because $t_{0}<t_{m}, s_{0}<f\left(t_{m}\right)$; and $g(\sigma)$ is a subset of either $\operatorname{cl}\left(f\left(t_{m}\right)\right)$ or $\operatorname{cl}\left(\left\langle f\left(v_{1}\right), b_{1}\right\rangle * s_{0}\right)$ or $\operatorname{cl}\left(f\left(v_{2}\right) * s_{0}\right)$. So by Lemma $3.3|g|$ is $\mathcal{U}$-homotopic to $|f|$ rel $|L|$.

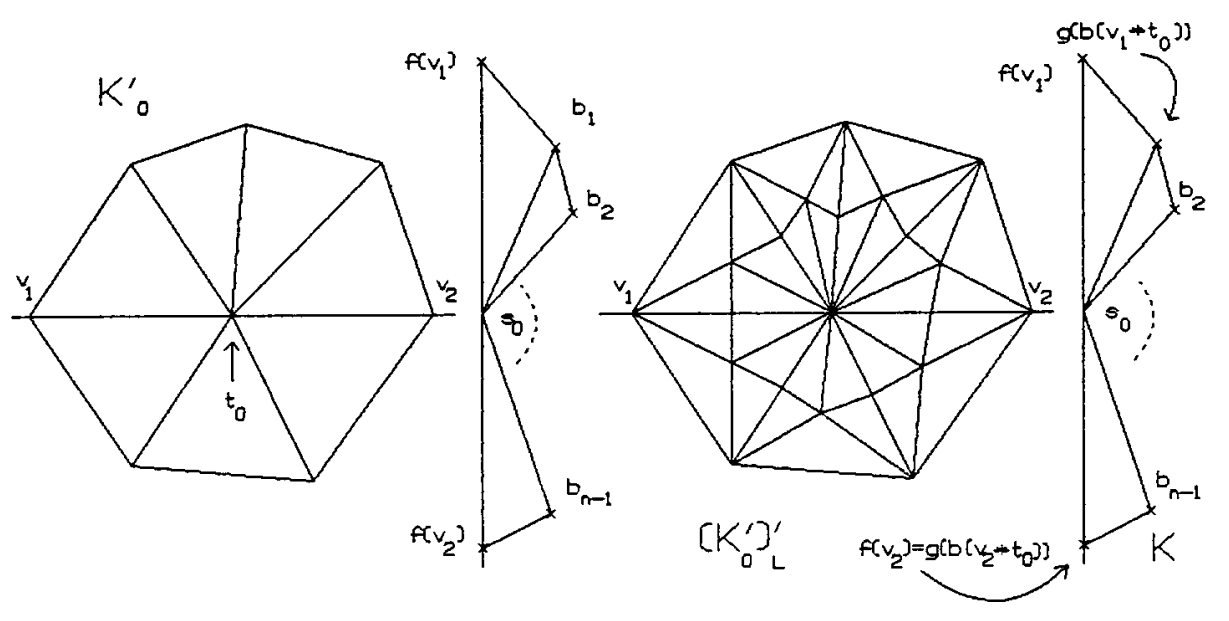

Figure 3.

Let $v_{1}^{\prime}=b\left(v_{1} * t_{0}\right)$ and $v_{2}^{\prime}=b\left(v_{2} * t_{0}\right)$. Then $l k\left(t_{0},\left(M_{s_{0}}\right)_{L}^{\prime}\right)$ consists of $v_{1}^{\prime}$ and $v_{2}^{\prime}$ and $c_{n-1} \equiv\left\langle g\left(v_{1}^{\prime}\right)\right\rangle \cup\left\langle g\left(v_{1}^{\prime}\right), b_{2}\right\rangle \cup \ldots \cup\left\langle b_{n-1}, g\left(v_{2}^{\prime}\right)\right\rangle \cup\left\langle g\left(v_{2}^{\prime}\right)\right\rangle$ is an arc in $l k\left(s_{0}, K\right)$ with endpoints $g\left(v_{1}\right)$ and $g\left(v_{2}^{\prime}\right)$ consisting of $n-11$ simplexes.

Proposition 3.7. Assume $t_{0}$ is a p-simplex of $K_{0}^{\prime}, t_{0}$ contains a fixed point of $|f|, f\left(t_{0}\right)=s_{0}$, and $\operatorname{dim}\left(s_{0}\right)=p$. Assume lk $\left(t_{0}, M_{s_{0}}^{\prime}\right)$ consists of two vertices $v_{1}$ and $v_{2}$ and that $f\left(v_{1}\right)$ and $f\left(v_{2}\right)$ are in the same component of $\left|l k\left(s_{0}, K\right)\right|$. Let $L$ be the subcomplex of $K_{0}^{\prime}$ consisting of all simplexes that do not have $t_{0}$ as a face. Then there is a simplicial map $|g|:\left|\left(K_{0}^{\prime}\right)_{L}^{\prime}\right| \rightarrow|K|$ 
$\mathcal{U}$-homotopic to $|f|$ rel $|L|$ such that $|g|$ has no fixed points in $t_{0}$.

Proof. Since $f\left(v_{1}\right)$ and $f\left(v_{2}\right)$ are in the same component of $\left|l k\left(s_{0}, K\right)\right|$, by using Lemma 3.6 repeatedly if necessary, assume $f\left(v_{1}\right)=f\left(v_{2}\right)$. Define $g$ on the vertices of $\left(K_{0}^{\prime}\right)_{L}^{\prime}$ as follows: If $a$ is a vertex of $L, g(a)=f(a)$; if $t_{0}$ is a proper face of $t$ and $t \neq v_{i} * t_{0}$ for $i=1$ or 2 then let $g(b(t))$ be any vertex of $s_{0}$; for $i=1$ and 2 let $g\left(b\left(v_{i} * t_{0}\right)\right)=f\left(v_{1}\right)$; let $g\left(b\left(t_{0}\right)\right)=f\left(v_{1}\right)$.

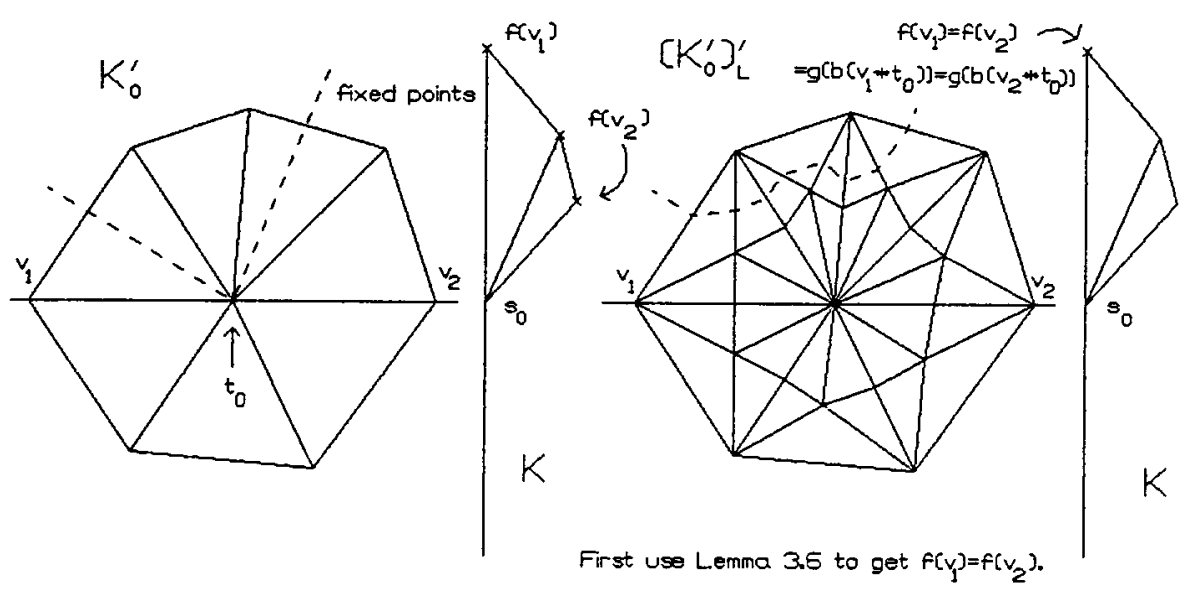

Figure 4.

Let $\sigma=\left\langle a_{0}, \ldots, a_{l}, b\left(t_{l+1}\right), \ldots, b\left(t_{m}\right)\right\rangle$ be a simplex of $\left(K_{0}^{\prime}\right)_{L}^{\prime}$. By definition, $g\left(a_{j}\right)$ is a vertex of $f\left(t_{l+1}\right)$ for $0 \leq j \leq l$, and, for $l+1 \leq j \leq$ $m, g\left(b\left(t_{j}\right)\right)=f\left(v_{1}\right)$ or a vertex of $s_{0}$. If for each $j, g\left(b\left(t_{j}\right)\right)$ is a vertex of $s_{0}$, then, since $s_{0}<f\left(t_{l+1}\right), g(\sigma)<f\left(t_{l+1}\right)$. If for some $j_{0}, g\left(b\left(t_{j_{0}}\right)\right)=f\left(v_{1}\right)$, then $g(\sigma)$ is a face of $f\left(v_{1}\right) * s_{0}$. In either case $g(\sigma)$ is a simplex of $K$. So $g$ : $\left(K_{0}^{\prime}\right)_{L}^{\prime} \rightarrow K$ is a simplicial map. Furthermore, $|f|(\sigma) \subset f\left(t_{m}\right), s_{0}<f\left(t_{m}\right)$, and $g(\sigma)$ is a subset of either $\operatorname{cl}\left(f\left(t_{m}\right)\right)$ or $\operatorname{cl}\left(f\left(v_{1}\right) * s_{0}\right)$. So by Lemma 3.3 $|g|$ is $\mathcal{U}$-homotopic to $|f|$ rel $|L|$.

Now $t_{0}=\cup \tau_{i}$ where each $\tau_{i}=\left\langle a_{0}, \ldots, a_{q_{i}}, b\left(t_{0}\right)\right\rangle$ is a simplex in $\left(K_{0}^{\prime}\right)_{L}^{\prime}$. Because $g\left(b\left(t_{0}\right)\right)$ is not a vertex of $s_{0}, g\left(\tau_{i}\right) \neq s_{0}$. Since $\tau_{i} \subset t_{0},|\pi|\left(\tau_{i}\right) \subset s_{0}$. So no point of $t_{0}$ can be a fixed point of $|g|$.

Proposition 3.8. Assume $t_{0}$ is a p-simplex of $K_{0}^{\prime}, t_{0}$ contains a fixed point of $|f|, f\left(t_{0}\right)=s_{0}$, and $\operatorname{dim}\left(s_{0}\right)=p$. Assume lk $\left(t_{0}, M_{s_{0}}\right)$ consists of just one vertex $v$ and that $f(v)$ is not a vertex of $s_{0}$. Let $L$ be the subcomplex of $K_{0}^{\prime}$ consisting of all simplexes that do not have $t_{0}$ as a face. Then there is a 
simplicial map $|g|:\left|\left(K_{0}^{\prime}\right)_{L}^{\prime}\right| \rightarrow|K| \mathcal{U}$-homotopic to $|f|$ rel $|L|$ such that $\left|t_{0}\right|$ contains no fixed points of $|g|$.

Proof. Define $g$ on the vertices of $\left(K_{0}^{\prime}\right)_{L}^{\prime}$ as follows: If $a$ is a vertex of $L$, $g(a)=f(a)$; if $t_{0}$ is a proper face of $t$ and $t \neq v * t_{0}$, then let $g(b(t))$ be any vertex of $s_{0}$; let $g\left(b\left(v * t_{0}\right)\right)=f(v)$ and let $g\left(b\left(t_{0}\right)\right)=f(v)$.
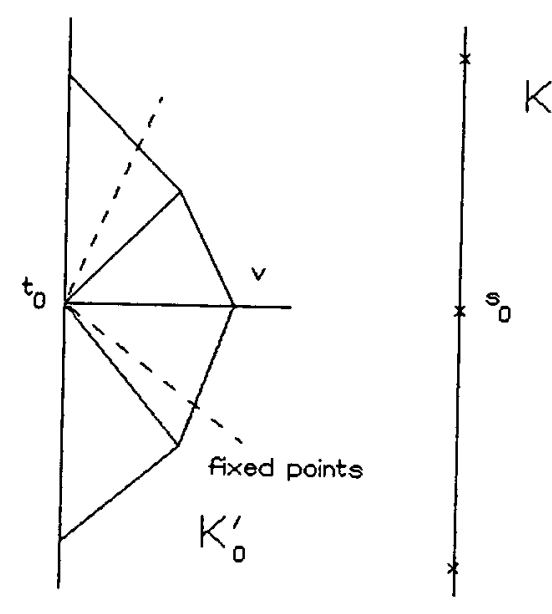
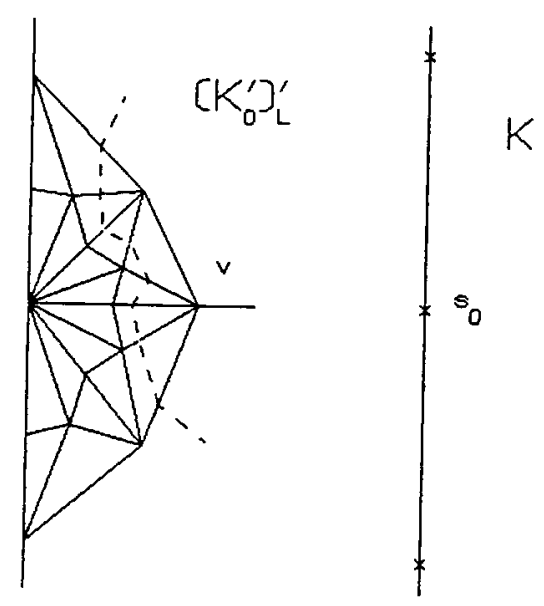

Figure 5.

Let $\sigma \in\left(K_{0}^{\prime}\right)_{L}^{\prime}$ be the simplex $\left\langle a_{0}, \ldots, a_{l}, b\left(t_{l+1}\right), \ldots, b\left(t_{m}\right)\right\rangle$. By definition, $g\left(a_{j}\right)$ is a vertex of $f\left(t_{l+1}\right)$ for $0 \leq j \leq l$, and, for $l+1 \leq j \leq$ $m, g\left(b\left(t_{j}\right)\right)=f(v)$ or is a vertex of $s_{0}$. If for each $j, g\left(b\left(t_{j}\right)\right)$ is a vertex of $s_{0}$, then, since $s_{0}<f\left(t_{l+1}\right), g(\sigma)<f\left(t_{l+1}\right)$. If for some $j_{0}, g\left(b\left(t_{j_{0}}\right)\right)=f(v)$, then $j_{0}=l+1$ and $t_{j_{0}}$ is the simplex $v * t_{0}$ or $t_{0}$. So $g(\sigma)<f(v) * s_{0}$. In all cases $f(\sigma)$ is a simplex of $K$ and so $g:\left(K_{0}^{\prime}\right)_{L}^{\prime} \rightarrow K$ is simplicial. Also $|f|(\sigma) \subset f\left(t_{m}\right), s<f\left(t_{m}\right)$, and $g(\sigma)$ is a subset of either $\operatorname{cl}\left(f\left(t_{m}\right)\right)$ or $\operatorname{cl}\left(s_{0} * f(v)\right)$, so by Lemma $3.3|g|$ is $\mathcal{U}$-homotopic to $|f|$ rel $|L|$.

The same proof as was used in Proposition 3.7 shows that $t_{0}$ contains no fixed points of $|g|$.

Proposition 3.9. Assume $t_{0}$ is a $(p+1)$-simplex of $K_{0}^{\prime}, t_{0}$ contains a fixed point of $|f|, f\left(t_{0}\right)=s_{0}, \operatorname{dim}\left(s_{0}\right)=p$, and $l k\left(s_{0}, K\right) \neq \emptyset$. Let $L$ be the subcomplex of $K_{0}^{\prime}$ consisting of all simplexes that do not have $t_{0}$ as a face. Then there is a simplicial map $|g|:\left|\left(K_{0}^{\prime}\right)_{L}^{\prime}\right| \rightarrow|K| \mathcal{U}$-homotopic to $|f|$ rel $|L|$ such that $t_{0}$ contains no fixed points of $|g|$.

Proof. Let $w_{0}$ be the vertex of $s_{0}$ that is the image under $f$ of two vertices of $t_{0}$ and let $b$ be a vertex in $l k\left(s_{0}, K\right)$. Define $g$ on the vertices of $\left(K_{0}^{\prime}\right)_{L}^{\prime}$ as 
follows: If $a$ is a vertex of $L, g(a)=f(a)$; if $t_{0}$ is a proper face of the simplex $t$ in $K_{0}^{\prime}$, then let $g(b(t))$ be the vertex $w_{0}$; let $g\left(b\left(t_{0}\right)\right)$ be the vertex $b$.

Let $\sigma=\left\langle a_{0}, \ldots, a_{l}, b\left(t_{l+1}\right), \ldots, b\left(t_{m}\right)\right\rangle$ be a simplex of $\left(K_{0}^{\prime}\right)_{L}^{\prime}$. By definition $g\left(a_{j}\right)$ is a vertex of $f\left(t_{l+1}\right)$ for $0 \leq j \leq l$, and, for $l+1 \leq j \leq m, g\left(b\left(t_{j}\right)\right)$ is either the vertex $b$ or the vertex $w_{0}$. If for each $j, g\left(b\left(t_{j}\right)\right)=w_{0}$, then, since $s_{0}<f\left(t_{l+1}\right), g(\sigma)<f\left(t_{l+1}\right)$. If for some $j_{0}, g\left(b\left(t_{j_{0}}\right)\right)=b$, then $j_{0}=l+1$ and $t_{j_{0}}=t_{0}$. So $g(\sigma)$ is a face of $b * s_{0}$. In either case $g(\sigma)$ is a simplex of $K$. So $g:\left(K_{0}^{\prime}\right)_{L}^{\prime} \rightarrow K$ is simplicial. Moreover, $|f|(\sigma) \subset f\left(t_{m}\right), s_{0}<f\left(t_{m}\right)$, and $g(\sigma)$ is a subset of either $\operatorname{cl}\left(f\left(t_{m}\right)\right)$ or $\operatorname{cl}\left(s_{0} * b\right)$, thus, by Lemma 3.3, $|g|$ is $\mathcal{U}$-homotopic to $|f|$ rel $|L|$.

Now $t_{0}=\cup \tau_{i}$ where each $\tau_{i}=\left\langle a_{0}, \ldots, a_{q_{i}} b\left(t_{0}\right)\right\rangle$ is a simplex in $\left(K_{0}^{\prime}\right)_{L}^{\prime}$. Because $g\left(b\left(t_{0}\right)\right)$ is not a vertex of $s_{0}, g\left(\tau_{i}\right) \neq s_{0}$. Since $\tau_{i} \subset t_{0},|\pi|\left(\tau_{i}\right) \subset s_{0}$. So $\tau_{i}$ contains no fixed points of $|g|$ and so no point of $t_{0}$ can be a fixed point of $|g|$.

Addendum 3.10. If $\sigma \in\left(K_{0}^{\prime}\right)_{L}^{\prime}$ contains a fixed point of $|g|,|\pi|(\sigma) \subset$ $s, \operatorname{dim}(s) \leq p$, and $\sigma$ is not a face of $t_{0}$, then $\sigma \in L$ and $s t\left(\sigma, K_{0}^{\prime}\right) \subset L$.

Note that if $t$ is a proper face of $t_{0}$, then, by definition of $L, t \in L$.

Proof. Let $\sigma$ be the simplex $\left\langle a_{0}, \ldots, a_{l}, b\left(t_{l+1}\right), \ldots, b\left(t_{q}\right)\right\rangle$ and suppose $q \neq$ l. Because $\sigma \subset t_{q}$ and $|\pi|(\sigma) \subset s,|\pi|\left(t_{q}\right) \subset s$. So $t_{0}<t_{q},|\pi|\left(t_{q}\right) \subset s$, and $|\pi|\left(t_{0}\right) \subset s_{0}$, by Lemma 3.1(a), $s_{0}<s$. Since $\operatorname{dim}(s) \leq \operatorname{dim}\left(s_{0}\right), s=s_{0}$. So $t_{q} \in M_{s_{0}}^{\prime}$. But $t_{0}$ is a principal simplex of $M_{s_{0}}^{\prime}$ and $t_{0}<t_{q}$, thus $t_{q}=t_{0}$ and $\sigma \subset t_{0}$. This contradicts the fact that $t_{0}$ contains no fixed points of $|g|$. Thus $q=l$ and $\sigma \in L$.

Suppose $\sigma * t_{0} \in K_{0}^{\prime}$. By Lemma 3.1(c), $s_{0}<s$ or $s<s_{0}$. Since $\operatorname{dim}(s) \leq$ $\operatorname{dim}\left(s_{0}\right), s<s_{0}$. By Lemma 3.1(b), $\sigma * t_{0} \in M_{s_{0}}^{\prime}$. Because $t_{0}$ is a principal simplex of $M_{s_{0}}^{\prime}$ and $t_{0}$ is a face of $t_{0} * \sigma, t_{0}=\sigma * t_{0}$. But this contradicts the fact that $\sigma$ is not a face of $t_{0}$. Thus $\sigma * t_{0} \notin K_{0}^{\prime}$. On the other hand if $t \in \operatorname{st}\left(\sigma, K_{0}^{\prime}\right)$ and $t_{0}<t$, then $t_{0} * \sigma \in K_{0}^{\prime}$. Thus $\operatorname{st}\left(\sigma, K_{0}^{\prime}\right) \subset L$.

Addendum 3.11. Assume $t \in\left(K_{0}^{\prime}\right)_{L}^{\prime}$ contains a fixed point of $|g|, t$ projects into the simplex $s$ of $K, \operatorname{dim}(s)<p, t<t_{0}$ and $\operatorname{Fix}(|f|) \cap\left|s t\left(t, K_{0}^{\prime}\right)\right|$ is a 1-manifold. If $t, K_{0}^{\prime}$, and $|f|$ satisfy Condition 1, then $t \in L, \operatorname{Fix}(|g|) \cap$ $\mid$ st $\left(t, K_{0}^{\prime}\right)_{L}^{\prime} \mid$ is a 1-manifold and $t,\left(K_{0}^{\prime}\right)_{L}^{\prime}$, and $g$ satisfy Condition 1 .

Proof. The simplexes of $s t\left(t, K_{0}^{\prime}\right)$ can be split into two sets $I_{1}=\left\{t^{\prime} \in K_{0}^{\prime} \mid t_{0}\right.$ is not a face of $\left.t^{\prime}\right\}$ and $I_{2}=\left\{t^{\prime} \in K_{0}^{\prime} \mid t_{0}<t^{\prime}\right\}$. By assumption Fix $(|f|) \cap$ $\left|s t\left(t, K_{0}^{\prime}\right)\right|$ is the union of two line segments: One of these is contained in $t_{0}$, hence the other line segment cannot meet any other simplex $t^{\prime} \in I_{2}$ since otherwise $t^{\prime} \cap \operatorname{Fix}(|f|)$ would be at least 2-dimensional. Thus the second line segment is contained in a simplex in $I_{1}$. On the other hand, st $\left(t,\left(K_{0}^{\prime}\right)_{L}^{\prime}\right)=I_{1} \cup I_{2}^{\prime}$ 
where $I_{2}^{\prime}=\left\{\left\langle v_{0}, v_{1}, \ldots, v_{q}, b\left(t_{q+1}\right), \ldots, b\left(t_{n}\right)\right\rangle \mid t=\left\langle v_{0}, \ldots, v_{q}\right\rangle\right\}$. Notice that $g=f$ on simplexes of $I_{1}$. It remains to find a simplex in $I_{2}^{\prime}$ containing a line segment in $\operatorname{Fix}(|g|)$ and to show that no other simplex in $I_{2}^{\prime}$ contains a fixed point.

Since $\operatorname{Fix}(|f|) \cap\left|s t\left(t, K_{0}^{\prime}\right)\right|$ is a 1 -manifold and $t_{0}$ contains a fixed point of $|f|$, a component, $A$, of $\left(\operatorname{Fix}(|f|) \cap\left|s t\left(t, K_{0}^{\prime}\right)\right|\right)-t$ is contained in $t_{0}$. By Theorem A this component is a line segment with one endpoint the fixed point in $t$ and the other endpoint a fixed point in a face $t^{\prime}$ of $t_{0}$. (Note that $t^{\prime} \neq t$ else $A \subset t$.) Note that $t^{\prime} * t=t_{0}$ because $t^{\prime} * t$ is a simplex of $K_{0}^{\prime}$ and $A \subset t^{\prime} * t$. By Lemma 2.4 there is a vertex $v$ of $t_{0}$ such that $|\pi|(v) \in s_{0}$. But $v$ is not a vertex of $t$, so, since $t^{\prime} * t=t_{0}, v$ must be a vertex of $t^{\prime}$. So by Lemma $2.5|\pi|\left(t^{\prime}\right) \subset s_{0}$. Since $t^{\prime}$ contains a fixed point of $|f|, f\left(t^{\prime}\right)=s_{0}$. Thus $t^{\prime}$ is a $p$-face of $t_{0}$ and $t^{\prime}$ contains a fixed point of $|f|$.

Let $t=\left\langle a_{0}, \ldots, a_{q}\right\rangle$ and $t_{0}=\left\langle a_{0}, \ldots, a_{q}, \ldots, a_{p+1}\right\rangle$. Now, all but one vertex $a_{i}$ of $t_{0}$ is a vertex of $t^{\prime}$. This vertex must be a vertex of $t$ since otherwise $t<t^{\prime}$ implies $t^{\prime} * t=t^{\prime} \neq t_{0}$. So $t^{\prime}=\left\langle a_{0}, \ldots, \hat{a}_{i}, \ldots, a_{q}, \ldots, a_{p+1}\right\rangle$.

Let $s_{1}=b * s_{0}$ and let $\gamma$ be the unique $(p+2)$-simplex in $M_{s_{1}}^{\prime}$ having $t_{0}$ as a face. Since $f\left(t^{\prime}\right)=s_{0}, f\left(a_{i}\right)=w_{0}$ and there is a vertex $a_{j}, j \neq i$ of $t^{\prime}$ such that $w_{0}=f\left(a_{j}\right)$. Let $\mu=\left\langle a_{0}, \ldots, \hat{a}_{i}, \ldots, a_{q}, \ldots, \hat{a}_{j}, \ldots, a_{p+1}, b\left(t_{0}\right), b(\gamma)\right\rangle$. Then $\mu$ is a $(p+1)$-simplex in $\left(K_{0}^{\prime}\right)_{L}^{\prime}$ contained in $\gamma, g(\mu)=b * s_{0},|\pi|(\mu) \subset$ $b * s_{0}$ and no proper face of $\mu$ contains a fixed point of $|g|$ so $\mu$ contains a fixed point of $|g|$. Thus $t * \mu$ contains a line segment of fixed points of $|g|$ with an endpoint in $t$.

Suppose $\sigma \in \operatorname{st}\left(t,\left(K_{0}^{\prime}\right)_{L}^{\prime}\right)$ contains a fixed point of $|g|$. Then $\sigma=$ $\left\langle v_{0}, v_{1}, \ldots, v_{r}, b\left(t_{r+1}\right), \ldots, b\left(t_{n}\right)\right\rangle$ where $\left\langle v_{0}, v_{1}, \ldots, v_{r}\right\rangle \in L, t_{r+1}, \ldots, t_{n} \notin L$ and $\left\langle v_{0}, v_{1}, \ldots, v_{r}\right\rangle<t_{r+1}<\cdots<t_{n}$. If $n=r$, then $\sigma=\left\langle v_{0}, v_{1}, \ldots, v_{n}\right\rangle \in L$ so $\sigma=t$ or $\sigma$ is a simplex of $K_{0}^{\prime}$ having $t$ as a proper face but not having $t_{0}$ as a face. Now suppose $n \neq r$ and let $g(\sigma)=s^{\prime}$. Because $\sigma \subset t_{n}$ and $|\pi|(\sigma) \subset s^{\prime},|\pi|\left(t_{n}\right) \subset s^{\prime}$. So $t_{n} \in M_{s^{\prime}}^{\prime}$. Since $t_{0}<t_{r+1}$ each vertex of $\left\langle v_{0}, v_{1}, \ldots, v_{r}\right\rangle$ is a vertex of $t_{0}$ or $l k\left(t_{0}, K_{0}^{\prime}\right)$, so by Condition $1 g\left(v_{i}\right)$ is a vertex of $s_{0}$. If $t_{0} \neq t_{r+1}$, then $g(\sigma)<s_{0}$. So $s^{\prime}<s_{0}$ and because $s_{0}$ is a $p$ simplex, $t_{n}$ is at most a $(p+1)$-simplex. But this contradicts the fact that the $(p+1)$-simplex $t_{0}$ is a proper face of $t_{n}$. Thus $t_{0}=t_{r+1}$ and so $g(\sigma)<b * s_{0}$. If $g(\sigma) \neq b * s_{0}$, then $\operatorname{dim}\left(t_{n}\right)$ is at most $p+1$. So $\sigma=\left\langle v_{0}, \ldots, v_{r}, b\left(t_{0}\right)\right\rangle$. But then $|\pi|(\sigma) \subset s_{0}$, yet $g(\sigma)$ has $b$ as a vertex. This contradicts the assumption that $\sigma$ contains a fixed point of $|g|$. So $g(\sigma)=b * s_{0}$ and $t_{n}$ is at most a $(p+2)$-simplex of $M_{b * s_{0}}^{\prime}$. Since $\gamma$ is the unique $(p+2)$-simplex in $M_{b * s_{0}}^{\prime}$ having $t_{0}$ as a face $t_{n}=\gamma$, so $\sigma=\left\langle v_{0}, v_{1}, \ldots, v_{r}, b\left(t_{0}\right), b(\gamma)\right\rangle$. Since $\left\langle v_{0}, \ldots, v_{r}\right\rangle<$ $t_{0}, \quad \sigma=\left\langle a_{0}, \ldots, a_{i}, \ldots, a_{q}, \ldots, a_{j}, \ldots, \hat{a}_{l}, \ldots, a_{p+1}, b\left(t_{0}\right), b(\gamma)\right\rangle . \quad$ [Remember that $f\left(a_{i}\right)=f\left(a_{j}\right)=f(b(\gamma))=w_{0}$.] Since $g(\sigma)=b * s_{0}, j=l$. So $\sigma=t * \mu$. Thus the only simplexes in $s t\left(t,\left(K_{0}^{\prime}\right)_{L}^{\prime}\right)$ containing fixed points 
are $t * \mu, t$, and simplexes $t_{i}$ in $K_{0}^{\prime}$ having $t$ as a face but not $t_{0}$ as a face. Since $\left|s t\left(t, K_{0}^{\prime}\right)\right| \cap \operatorname{Fix}(|f|)$ is a 1-manifold at most one such $t_{i}$ exists. Thus $\left|s t\left(t,\left(K_{0}^{\prime}\right)_{L}^{\prime}\right)\right| \cap \operatorname{Fix}(|g|)$ is a 1-manifold.

To see that $\left(K_{0}^{\prime}\right)_{L}^{\prime}, g$, and $t$ satisfy Condition 1 , suppose $v$ is a vertex in $l k\left(t * \mu,\left(K_{0}^{\prime}\right)_{L}^{\prime}\right)$. Then $v=a_{j}$ or $v=b\left(t_{r}\right)$ where $t_{r} \in K_{0}^{\prime}$ and $t_{0}<t_{r}$. By definition of $g, g(v)$ is a vertex of $s_{0} * b$.

The next lemma holds true for the construction of $\left(K_{0}^{\prime}\right)_{L}^{\prime}$ and $g$ as given in Propositions 3.4, 3.7 and 3.8 and Lemma 3.6. The proof is given for the construction in Proposition 3.4 but is similar, almost word for word, for the other constructions of $g$ and $\left(K_{0}^{\prime}\right)_{L}^{\prime}$.

Lemma 3.12. If $\sigma \neq t_{0}$ is a simplex of $\left(K_{0}^{\prime}\right)_{L}^{\prime}$ and $\sigma$ contains a fixed point of $|g|$ and $\sigma$ projects into a simplex $s$ of $K$ of dimension $\leq p$, then $\sigma$ is also a simplex of $K_{0}^{\prime}$; furthermore $s t\left(\sigma, K_{0}^{\prime}\right)_{L}^{\prime} \subset K_{0}^{\prime}$.

Proof. Let $\sigma=\left\langle a_{0}, \ldots a_{l}, b\left(t_{l+1}\right), \ldots, b\left(t_{q}\right)\right\rangle$ and suppose $q \neq l$. Because $\sigma \subset t_{q},|\pi|\left(t_{q}\right) \subset s$. Also $t_{0}<t_{q}$ and $|\pi|\left(t_{0}\right) \subset s_{0}$, so by Lemma 3.1(a), $s_{0}<s$. Since $\operatorname{dim}(s) \leq \operatorname{dim}\left(s_{0}\right), s=s_{0}$. So $t_{q} \in M_{s_{0}}^{\prime}$. But $t_{0}$ is a face of exactly three simplexes of $M_{s_{0}}^{\prime}$, namely $t_{0}, v_{1} * t_{0}$, and $v_{2} * t_{0}$. So $\sigma=\left\langle a_{0}, \ldots a_{l}, b\left(v_{i} * t_{0}\right)\right\rangle$ for $i=1$ or 2 . But $|\pi|(\sigma) \subset s_{0}$ yet $g(\sigma) \neq s_{0}$ because $g\left(b\left(v_{i} * t_{0}\right)\right)$ is not a vertex of $s_{0}$ for $i=1$ or 2 . This contradicts the fact that $\sigma$ contains a fixed point of $|g|$. Thus $q=l$ and $\sigma \in L$.

Suppose $\sigma * t_{0} \in K_{0}^{\prime}$. By Lemma 3.1(c) $s_{0}<s$ or $s<s_{0}$. Since $\operatorname{dim}(s) \leq$ $\operatorname{dim}\left(s_{0}\right), s<s_{0}$. So by Lemma 3.1(b) $\sigma * t_{0} \in M_{s_{0}}^{\prime}$. Since $t_{0}<\sigma * t_{0}, \sigma * t_{0}=$ $v_{1} * t_{0}$ or $v_{2} * t_{0}$ or $t_{0}$. Since both $\sigma$ and $t_{0}$ contain a fixed point of $|f|, \sigma * t_{0}$ contains a line segment of fixed points of $|f|$. By Theorem A $\sigma * t_{0} \neq t_{0}$. Since $|\pi|\left(v_{i} * t_{0}\right) \subset s_{0}$ and $f\left(v_{i}\right)$ is not a vertex of $s_{0}$ for $i=1$ or 2 , the simplexes $v_{1} * t_{0}$ and $v_{2} * t_{0}$ do not contain fixed points of $|f|$, thus $\sigma * t_{0} \neq v_{1} * t_{0}$ or $v_{2} * t_{0}$. This means $\sigma * t_{0} \notin K_{0}^{\prime}$ or $\sigma=t_{0}$.

Now if $t$ is a simplex in $K_{0}^{\prime}$ and $\sigma \neq t_{0}$ and $\sigma<t$ and $t_{0}<t$, then $t_{0} * \sigma<t$ which contradicts the fact that $t_{0} * \sigma \notin K_{0}^{\prime}$; so if $t \in \operatorname{st}\left(\sigma, K_{0}^{\prime}\right)$, then $t_{0}$ is not a face of $t$ so $t \in L$.

Proposition 3.13. There is a subdivision $K_{0}^{\prime \prime}$ of $K_{0}^{\prime}$ and a simplicial map $|g|:\left|K_{0}^{\prime \prime}\right| \rightarrow|K| \mathcal{U}$-homotopic to $|f|$ such that

(i) if $t$ is a $(p+1)$-simplex of $K_{0}^{\prime \prime}$ and $t$ projects into a $p$-simplex $s$ of $K$ and $t$ contains a fixed point of $|g|$, then $l k(s, K)=\emptyset$,

(ii) if $t$ is a p-simplex of $K_{0}^{\prime \prime}$ and $t$ projects into a p-simplex $s$ of $K$ and $t$ contains a fixed point of $|g|$ and $l k(s, K) \neq \emptyset$, then

(a) $l k(s, K)$ is not connected,

(b) $\operatorname{Fix}(|g|) \cap\left|s t\left(t, K_{0}^{\prime \prime}\right)\right|$ is a 1-manifold, 
(c) Condition 1 is satisfied by $t, K_{0}^{\prime \prime}$, and $g$.

Proof. We work by induction on the skeleta of $K$.

Assume there is a subdivision $K_{0}^{\prime \prime}$ of $K_{0}^{\prime}$ and a simplicial map $|g|:\left|K_{0}^{\prime \prime}\right| \rightarrow$ $|K| \mathcal{U}$-homotopic to $|f|$ such that over the $(q-1)$-skeleton of $K$, statements (i) and (ii) hold. The induction starts trivially with the (empty) (-1)-skeleton.

If $t_{0}$ is a $(q+1)$-simplex of $K_{0}^{\prime \prime}$ and $t_{0}$ projects into the $q$-simplex $s_{0}$ of $K$ and $t_{0}$ contains a fixed point of $|g|$ and $l k\left(s_{0}, K\right) \neq \emptyset$, then by Proposition 3.9, there is a subdivision $\left(K_{0}^{\prime \prime}\right)^{\prime}$ of $K_{0}^{\prime \prime}$ and a simplicial map $\left|g^{\prime}\right|:\left|\left(K_{0}^{\prime \prime}\right)^{\prime}\right| \rightarrow|K|$ homotopic to $|g|$ such that $t_{0}$ contains no fixed points of $\left|g^{\prime}\right|$. By Addendum 3.10 if $t$ is a simplex of $K_{0}^{\prime \prime}$ and $t$ contains a fixed point of $\left|g^{\prime}\right|$ and $t$ projects into the $q$-skeleton of $K$, then $t$ is also a simplex of $K_{0}^{\prime}$. By definition of $\left|g^{\prime}\right|$, $|g|$ and $\left|g^{\prime}\right|$ agree on $t$. Thus if $x$ is a fixed point of $\left|g^{\prime}\right|$ and $x$ projects into the $q$-skeleton of $K$, then $x$ is a fixed point of $|g|$. So no new fixed points are created over the $q$-skeleton of $K$.

Furthermore, suppose $t$ is a $p$-simplex of $\left(K_{0}^{\prime \prime}\right)^{\prime}, p \leq q-1$, and $t$ contains a fixed point of $\left|g^{\prime}\right|$. If $t$ is not a face of $t_{0}$ then by Addendum 3.10 each simplex of $s t\left(t,\left(K_{0}^{\prime \prime}\right)^{\prime}\right)$ is also a simplex of $K_{0}^{\prime \prime}$. So if $\operatorname{Fix}(|g|) \cap\left|s t\left(t, K_{0}^{\prime \prime}\right)\right|$ is a 1-manifold, then $\operatorname{Fix}\left(\left|g^{\prime}\right|\right) \cap\left|s t\left(t,\left(K_{0}^{\prime \prime}\right)^{\prime}\right)\right|$ is a 1-manifold; if Condition 1 is satisfied by $t, K_{0}^{\prime \prime}$ and $g$, then Condition 1 is satisfied by $t,\left(K_{0}^{\prime \prime}\right)^{\prime}$ and $g^{\prime}$. And if $t$ is a face of $t_{0}$, then, by Addendum 3.11, $\operatorname{Fix}\left(\left|g^{\prime}\right|\right) \cap\left|s t\left(t,\left(K_{0}^{\prime \prime}\right)^{\prime}\right)\right|$ is a 1-manifold and $t,\left(K_{0}^{\prime \prime}\right)^{\prime}$, and $g^{\prime}$ satisfy Condition 1 . Apply Proposition 3.9 to each $(q+1)$-simplex $t$ of $K_{0}^{\prime \prime}$ such that $t$ projects into a $q$-simplex $s$ of $K$, $t$ contains a fixed point of $|g|$ and $l k(s, K) \neq \emptyset$, subdividing the previously defined $K_{0}^{\prime \prime}$ each time. After a finite number of applications of Proposition 3.9 , there is a subdivision $K_{0}^{\prime \prime}$ of $K_{0}^{\prime}$ and a simplicial map $|g|:\left|K_{0}^{\prime \prime}\right| \rightarrow|K|$ $\mathcal{U}$-homotopic to $|f|$ such that for $p=0,1, \ldots, q$ statement (i) holds and for $p=0,1, \ldots, q-1$ statement (ii) holds.

Now suppose $t_{0}$ is a $q$-simplex of $K_{0}^{\prime \prime}$ and $t_{0}$ projects into the $q$-simplex $s_{0}$ of $K$ and $t_{0}$ contains a fixed point of $|g|$. If $v$ is a vertex in $l k\left(t_{0}, M_{s_{0}}^{\prime}\right)$ and $g(v)$ is a vertex of $s_{0}$, then the $(q+1)$-simplex $v * t_{0}$ contains a line segment of fixed points of $|g|$ and so $l k\left(s_{0}, K\right)=\emptyset$. Thus if $l k\left(s_{0}, K\right) \neq \emptyset$, then $g\left(l k\left(t_{0}, K_{0}^{\prime \prime}\right)\right) \subset l k\left(s_{0}, K\right)$. If $g\left(l k\left(t_{0}, K_{0}^{\prime \prime}\right)\right)$ is contained in a component of $\left|l k\left(s_{0}, K\right)\right|$ then, either by Proposition 3.7 or by Proposition 3.8, there is a subdivision $\left(K_{0}^{\prime \prime}\right)^{\prime}$ of $K_{0}^{\prime \prime}$ and a simplicial map $\left|g^{\prime}\right|:\left|\left(K_{0}^{\prime \prime}\right)^{\prime}\right| \rightarrow|K| \mathcal{U}$ homotopic to $|g|$ such that $t_{0}$ contains no fixed points of $\left|g^{\prime}\right|$. By Lemma 3.12, if $t$ is a simplex of $\left(K_{0}^{\prime \prime}\right)^{\prime}$ and $t$ contains a fixed point of $\left|g^{\prime}\right|$ and $t$ projects into a simplex of dimension $\leq q$, then $t$ corresponds to a simplex of $K_{0}^{\prime \prime}$ and each simplex in $s t\left(t, K_{0}^{\prime \prime}\right)$ corresponds to a simplex of $K_{0}^{\prime}$. Since $|g|$ and $\left|g^{\prime}\right|$ agree on $s t\left(t, K_{0}^{\prime \prime}\right)$, no new fixed points are added over the $q$-skeleton of $K$ and statement (ii) holds for $p=0,1, \ldots q-1$. Apply either Proposition 3.7 
or Proposition 3.8 to each $q$-simplex $t$ of $K_{0}^{\prime \prime}$ such that $t$ projects into a $q$ simplex $s$ of $K, t$ contains a fixed point of $|g|$ and $g\left(l k\left(t, M_{s}^{\prime}\right)\right)$ is contained in a component of $|l k(s, K)|$, subdividing the previously defined $\left(K_{0}^{\prime \prime}\right)^{\prime}$ each time. Thus there is a subdivision $K_{0}^{\prime \prime}$ of $K_{0}^{\prime}$ and a simplicial map $|g|:\left|K_{0}^{\prime \prime}\right| \rightarrow|K| \mathcal{U}$ homotopic to $|f|$ such that statement (i) holds for $p=0,1, \ldots, q$, statement (ii) holds for $p=0,1, \ldots, q-1$, and if $t$ is a $q$-simplex of $K_{0}^{\prime \prime}$ and $t$ projects into a $q$-simplex $s$ of $K$ and $t$ contains a fixed point of $|g|$ and $l k(s, K) \neq \emptyset$, then $l k\left(t, M_{s}^{\prime}\right)$ consists of two vertices $v_{1}$ and $v_{2}$ and $g\left(v_{1}\right)$ and $g\left(v_{2}\right)$ are in different components of $l k(s, K)$.

If $t_{0}$ is a $q$-simplex of $K_{0}^{\prime \prime}$ and $t_{0}$ projects into a $q$-simplex $s_{0}$ of $K$ and $t_{0}$ contains a fixed point of $|g|$ and $l k\left(s_{0}, K\right) \neq \emptyset$ but not connected, then by Proposition 3.4 there is a subdivision $\left(K_{0}^{\prime \prime}\right)^{\prime}$ of $K_{0}^{\prime \prime}$ and a simplicial map $\left|g^{\prime}\right|:\left|\left(K_{0}^{\prime \prime}\right)^{\prime}\right| \rightarrow|K|$ homotopic to $|g|$ such that $\operatorname{Fix}\left(\left|g^{\prime}\right|\right) \cap\left|s t\left(t_{0},\left(K_{0}^{\prime \prime}\right)^{\prime}\right)\right|$ is a 1-manifold. By Addendum 3.5, Condition 1 is satisfied by $v_{0},\left(K_{0}^{\prime \prime}\right)^{\prime}$ and $g^{\prime}$. By Lemma 3.12, if $t \neq t_{0}$ is a simplex of $\left(K_{0}^{\prime \prime}\right)^{\prime}$ and $t$ projects into a simplex $s$ of $K$ of dimension $\leq q$ and $t$ contains a fixed point of $\left|g^{\prime}\right|$, then $t$ is also a simplex of $K_{0}^{\prime \prime}$. Since $\left|g^{\prime}\right|$ and $|g|$ agree on $t$, no new fixed points have been added. Furthermore each simplex in $s t\left(t,\left(K_{0}^{\prime \prime}\right)^{\prime}\right)$ is also a simplex in $K_{0}^{\prime \prime}$. By definition of $\left|g^{\prime}\right|,\left|g^{\prime}\right|$ and $|g|$ agree on $\left|s t\left(t,\left(K_{0}^{\prime \prime}\right)^{\prime}\right)\right|$. So if $\operatorname{dim}(t)=\operatorname{dim}(s)$ and $\operatorname{Fix}(|g|) \cap\left|s t\left(t, K_{0}^{\prime \prime}\right)\right|$ is a 1-manifold then $\operatorname{Fix}\left(\left|g^{\prime}\right|\right) \cap\left|s t\left(t,\left(K_{0}^{\prime \prime}\right)^{\prime}\right)\right|$ is a 1-manifold. If Condition 1 is satisfied by $t, K_{0}^{\prime \prime}$ and $g$, then Condition 1 is satisfied by $t,\left(K_{0}^{\prime \prime}\right)^{\prime}$ and $g^{\prime}$.

Apply Proposition 3.4 to each $q$-simplex $t$ of $K_{0}^{\prime \prime}$ such that $t$ projects into a $q$-simplex $s$ of $K, t$ contains a fixed point of $|g|$ and $l k(s, K) \neq \emptyset$ but not connected, subdividing the previously defined $\left(K_{0}^{\prime \prime}\right)^{\prime}$ each time.

So there is a subdivision $K_{0}^{\prime \prime}$ of $K_{0}^{\prime}$ and a simplicial map $|g|:\left|K_{0}^{\prime \prime}\right| \rightarrow|K|$ $\mathcal{U}$-homotopic to $|f|$ such that for $p=0,1, \ldots, q$ statements (i) and (ii) hold. Thus, by induction there is a subdivision $K_{0}^{\prime \prime}$ of $K_{0}^{\prime}$ and a simplicial map $|g|$ $\mathcal{U}$-homotopic to $|f|$ satisfying (i) and (ii).

Proof of Theorem B. Let $K_{0}^{\prime \prime}$ and $g: K_{0}^{\prime \prime} \rightarrow K$ be the subdivision and map given by Proposition 3.13. Let $t$ be a simplex of $K_{0}^{\prime \prime}$, let $|\pi|(t) \subset s$ in $K$ and suppose $t$ contains a fixed point of $|g|$. By Theorem A either $\operatorname{dim}(t)=$ $\operatorname{dim}(s)$ or $\operatorname{dim}(t)=\operatorname{dim}(s)+1$. If $\operatorname{dim}(t)=\operatorname{dim}(s)+1$, by Proposition 3.13, $l k(s, K)=\emptyset$. If $\operatorname{dim}(t)=\operatorname{dim}(s)$ but $l k(s, K) \neq \emptyset$, then, by Proposition 3.13, $l k(s, K)$ is not connected. So $\operatorname{Fix}(|g|) \subset S_{0} \cup S_{1}$. If $\operatorname{dim}(t)=\operatorname{dim}(s)+1$, then by Theorem A, $t$ contains a line segment of fixed points with endpoints in $\operatorname{bd}(t)$, also $s t\left(t, K_{0}^{\prime \prime}\right)=\{t\}$. If $\operatorname{dim}(t)=\operatorname{dim}(s)$, then, by Theorem $\mathrm{A}, t$ contains exactly one fixed point. Suppose $\operatorname{dim}(t)=\operatorname{dim}(s)$ and $l k(s, K)=\emptyset$. Because $\operatorname{dim}(t)=\operatorname{dim}(s), l k\left(t, M_{s}^{\prime \prime}\right)$ consists of one or two vertices $v$. Because $g(v * t)$ is a simplex of $K$ having $s$ as a face and $l k(s, K)=\emptyset, g(v)$ is a vertex 
of $s$. So $v * t$ contains a line segment of fixed points of $|g|$ with one endpoint in $t$. So $\operatorname{Fix}(|g|) \cap\left|s t\left(t, K_{0}^{\prime \prime}\right)\right|$ is a 1-manifold or half-open line segment with endpoint in $t$ and $t \in \partial M_{s}^{\prime \prime}$. Thus $\operatorname{dim}\left(S_{1} \cap \operatorname{Fix}(|g|)\right)=1$. Suppose $\operatorname{dim}(t)=$ $\operatorname{dim}(s)$ but $l k(s, K) \neq \emptyset$. Since $l k(s, K) \neq \emptyset$, by Proposition 3.13, no simplex in $s t\left(t, M_{s}^{\prime \prime}\right)$ other than $t$ contains a fixed point of $|g|$. So $\operatorname{dim}\left(\operatorname{Fix}(|g|) \cap S_{0}\right)=$ 0 and by Proposition 3.13(ii) (b) $\operatorname{Fix}(|g|) \cap\left|s t\left(t, K_{0}^{\prime \prime}\right)\right|$ is a 1-manifold.

The reader will observe that our method achieves the claims in the final paragraph of Theorem B.

\section{Proof of Theorem C.}

Lemma 4.1. Let $U^{n+1}$ and $V^{n+1}$ be linear subspaces of the real vector space $W^{2 n+1}$ and let $U \cap V$ have dimension 1 . Then $(W, U, V, U \cap V) \cong$ $\left(\mathbb{R}^{2 n+1} ; \mathbb{R}^{n+1} \times 0,0 \times \mathbb{R}^{n+1}, 0^{n} \times \mathbb{R} \times 0^{n}\right)$.

Proof. Pick a basis, starting in $U \cap V$, extending to $U$ and also to $V$, then to $W$.

Lemma 4.2. If in addition $T^{2 n} \leq W^{2 n+1}$ is such that $\operatorname{dim}(U \cap T)=$ $\operatorname{dim}(V \cap T)=n$ and $\operatorname{dim}(U \cap V \cap T)=0$, then $(W, T, U, V, U \cap V) \cong$ $\left(\mathbb{R}^{2 n+1} ; \mathbb{R}^{n} \times 0 \times \mathbb{R}^{n}, \mathbb{R}^{n+1} \times 0,0 \times \mathbb{R}^{n+1}, 0 \times \mathbb{R} \times 0\right)$.

Proof. $U \cap V \cap T=\{0\}$. Start with a basis in $U \cap V$, extend to $(U \cap V) \dot{+}$ $(U \cap T) \dot{+}(U \cap T)$.

Lemma 4.2 implies:

Lemma 4.3. Let $W_{+}$be the closed half space on one side of $T$. Then $\left(W_{+}, T, U_{+}, V_{+},(U \cap V)_{+}\right) \cong\left(\mathbb{R}^{n} \times \mathbb{R}_{+} \times \mathbb{R}^{n} ; \mathbb{R}^{n} \times 0 \times \mathbb{R}^{n}, \mathbb{R}^{n} \times \mathbb{R}_{+} \times 0,0 \times\right.$ $\left.\mathbb{R}_{+} \times \mathbb{R}^{n}, 0 \times \mathbb{R}_{+} \times 0\right)$.

Proof of Theorem C. Let the PL manifold $|K|$ have dimension $n$. It follows from Theorem B that Fix $(|g|)$ consists of straight-line segments each of which connects two points in the interiors of $n$-dimensional faces of an $(n+1)$-simplex of $K_{0}^{\prime \prime}$; see Figure 6 . Transversality over a neighborhood of $a$ follows from Lemma 4.3, and over a neighborhood of $b$ from Lemma 4.1. Transversality over a neighborhood of $c$ also follows from Lemma 4.3, for if we have another $\left(W_{-}, T, \tilde{U}_{-}, \tilde{V}_{-},(\tilde{U} \cap \tilde{V})_{-}\right) \cong\left(\mathbb{R}^{n} \times \mathbb{R}_{-} \times \mathbb{R}^{n}, \mathbb{R}^{n} \times 0 \times\right.$ $\left.\mathbb{R}^{n}, \mathbb{R}^{n} \times \mathbb{R}_{-} \times 0,0 \times \mathbb{R}_{-} \times \mathbb{R}^{n}, 0 \times \mathbb{R}_{-} \times 0\right)$ we can piece the two together to get a PL homeomorphism $\left(W, T, U_{+} \cup \tilde{U}_{-}, V_{+} \cup \tilde{V}_{-},(U \cap V)_{+} \cup(\tilde{U} \cap \tilde{V})_{-}\right) \cong$ $\left(\mathbb{R}^{2 n+1} ; \mathbb{R}^{n} \times 0 \times \mathbb{R}^{n}, \mathbb{R}^{n+1} \times 0,0 \times \mathbb{R}^{n+1}, 0 \times \mathbb{R} \times 0\right)$. Thus the graph of $|g|$ is transverse to the graph of $|\pi|$. 


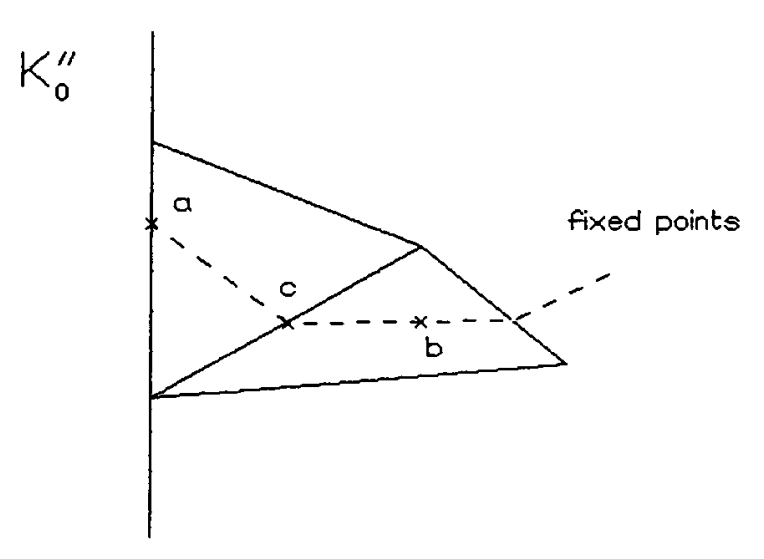

Figure 6.

\section{References}

[Br] R.F. Brown, The Lefschetz Fixed Point Theorem, Scott Foresman, Chicago 1971.

$\left[\mathrm{D}_{1}\right]$ D. Dimovski, One-Parameter Fixed Point Indices for Periodic Orbits, in Proceedings of the AMS-IMS-SIAM Joint Summer Research Conference on Nielsen Theory and Dynamical Systems Edited by C. McCord, 1993.

$\left[\mathrm{D}_{2}\right]$ - One-Parameter Fixed Point Indices, Pacific J. Math., 164 (1994), 263-297.

[DG] D. Dimovski and R. Geoghegan, One-Parameter Fixed Point Theory, Forum. Math., 2 (1990), 125-154.

$\left[\mathrm{GN}_{1}\right]$ R. Geoghegan and A. Nicas, Parametrized Lefschetz Nielsen Fixed Point Theory and Hochschild Homology, Trans. Amer. Math. Soc., 116 (1994), 397-446.

$\left[\mathrm{GN}_{2}\right]$ - Trace and Torsion in the Theory of Flows, Topology, 33 (1994), 683-719.

$\left[\mathrm{GN}_{3}\right] \longrightarrow$, Higher Euler Characteristic I, L'Enseignement Mathématique, 41 (1995), $3-62$.

$\left[\mathrm{GN}_{4}\right]$, Lefschetz Trace Formulae, Zeta Functions and Torsion in Dynamics, in Proceedings of the AMS-IMS-SIAM Joint Summer Research Conference on Nielsen Theory and Dynamical Systems Edited by C. McCord, 1993.

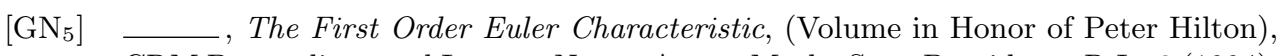
CRM Proceedings and Lecture Notes, Amer. Math. Soc., Providence R.I., 6 (1994), 37-59.

[GNO] R. Geoghegan, A. Nicas and G. Oprea, Higher Lefschetz Traces and Spherical Euler Characteristics, Trans. Amer. Math. Soc., in press.

[J] J. Jezierski, One-Codimensional Wecken Type Theorems, Forum Math., 5 (1993), 421-439. 
[RS] C.P. Rourke and B.J. Sanderson, Piecewise Linear Topology, Springer Verlag, New York, Heidelberg, Berlin, 1972.

[S] E. Spanier, Algebraic Topology, McGraw-Hill, New York, 1966.

Received November 20, 1994 and revised February 19, 1996. This is a chapter of the author's Ph.D. dissertation written under the direction of Ross Geoghegan to be presented at the State University of New York at Binghamton.

Binghamton University

Binghamton, NY 13902-6000

E-mail address: plavcha@ibm.net 